\title{
Site Response Implications Associated with using Non-Unique Vs Profiles from Surface Wave Inversion in Comparison with Other Commonly Used Methods of Accounting for Vs Uncertainty
}

\author{
David P. Teague ${ }^{\mathrm{a}}$, Brady R. Cox ${ }^{\mathrm{a}^{*}}$ \\ ${ }^{a}$ Department of Civil, Architectural and Environmental Engineering, The University of Texas, 301 E Dean Keeton \\ Stop C1792, Austin, TX, USA 78712 \\ * Corresponding author. Tel.: (512) 471-9162, Email address: brcox@utexas.edu
}

\begin{abstract}
$\underline{\text { Abstract }}$
This paper discusses variability and accuracy of site response predictions performed using shear wave velocity (Vs) profiles derived from non-unique surface wave inversions and other commonly used statistical methods of accounting for epistemic uncertainty and aleatory variability in Vs. Specifically, linear and equivalent linear site response analyses were performed on the following three classes of Vs profiles: (1) 350 Vs profiles developed by performing multiple surface wave inversions, each with a unique set of layering parameters, on a common dispersion dataset, (2) two upper/lower range base-case Vs profiles developed by systematically increasing or decreasing the solution Vs profile by 20\%, and (3) 100 Vs profiles developed using the Vs randomization procedure proposed by Toro (1995). Vs profiles derived from surface wave inversions generally yielded accurate site response estimates with minimal variability, so long as their theoretical dispersion data fit the experimental dispersion data well. On the other hand, the upper/lower range and randomized Vs profiles generally produced inaccurate and highly variable site response predictions, although the inclusion of site-specific parameters in the randomization model improved the results. At real sites where substantial aleatory variability is anticipated and/or the epistemic uncertainty is quite high, the site response estimates associated with the randomized and/or upper/lower range Vs profiles may be deemed acceptable. However, if the experimental dispersion data and horizontal-to-vertical spectral ratios are shown to be consistent over the footprint of a site, it may be possible to significantly reduce the uncertainty associated with the input Vs profile and the resulting uncertainty in the site response.
\end{abstract}

\section{$\underline{\text { Introduction }}$}

Site response simulations using equivalent linear and nonlinear analyses have shown that the shear wave velocity (Vs) profile selected to anchor small-strain subsurface stiffness conditions has a large influence on the amplitude and frequency content of predicted surface ground motions (e.g., Bazurro and Cornell 2004 [1], Rathje et al. 2010 [2], Li and Assimaki 2010 [3], Barani et al. 2013 [4]). Hence, the development of appropriate Vs profiles for use in site response analyses is of paramount importance. Engineering design codes stress the importance of accounting for uncertainty in Vs when performing site response analyses (e.g., ASCE 2010 [5], AASHTO 2011 [6]), yet, no firm guidelines are provided regarding how to appropriately/realistically account for these uncertainties.

Both epistemic uncertainty and aleatory variability in Vs are typically accounted for in probabilistic site response analyses for critical projects such as nuclear facilities (EPRI 2012 [7]). 
Epistemic uncertainty is accounted for by considering multiple base-case Vs profiles. Typically, a "mean" and upper/lower range base-cases are developed. When multiple Vs profiles are available for a given site, the "mean" base-case may be computed as the average (or median) of the available Vs profiles. However, oftentimes only a single Vs profile is available and assumed to represent the mean. The upper/lower range base-cases are developed by applying an estimated level of epistemic uncertainty to the "mean" base-case Vs profile. Oftentimes, upper/lower range base-cases are generated by arbitrarily increasing and decreasing the reference Vs profile by a constant factor such as $+/-20 \%$ to $30 \%$. Aleatory variability in Vs is accounted for in site response analyses via a randomization process about the base-case Vs profiles. This is most commonly performed using the Toro (1995) Vs randomization model. If abundant Vs data is available at a site, the statistical parameters needed to constrain epistemic uncertainty and aleatory variability can be obtained. Otherwise, conservative estimates must be made (Griffiths et al. 2016a [8]).

Vs profiles can be measured in-situ using invasive or non-invasive techniques. In either case, there is uncertainty associated with the final Vs profiles, which may or may not be openly acknowledged to the end-user. While it is commonly assumed that there is less uncertainty associated with invasive/borehole methods, a recent, comprehensive, blind-analysis study at three geologically-distinct sites in Europe revealed that Vs profiles derived from surface wave testing had coefficients of variation that were similar to, and at times lower than, those derived from a combination of crosshole, downhole and suspension logging (Garafalo et al. 2016a [9], Garafalo et al. 2016b [10]). Nonetheless, when Vs profiles are derived from an inversion of surface wave data, one must acknowledge that the solution is non-unique (Lai et al. 2005 [11], Cornou et al. 2009 [12], Cox et al. 2014 [13], Garafalo et al. 2016a [9]), and the uncertainties may be significant, particularly if results are reported without performing a systematic, rigorous investigation of different trial subsurface layering models (DiGiulio et al. 2012 [14], Cox and Teague 2016 [15]).

The surface wave inversion process involves finding one or more layered earth models whose theoretical dispersion curve(s) fit the experimentally-measured dispersion data. While in the past it was common to simply seek a solution that yielded a "reasonable" fit by-eye, it is presently common to quantify the quality of fit using some sort of a least-squares misfit value, with lower misfit values indicating a better fit. Layered earth models are comprised of a system of stacked, linear elastic, horizontal layers over a half-space. Each layer is defined by its inversion parameters: thickness $(\mathrm{t})$, shear wave velocity (Vs), compression wave velocity (Vp) or Poisson's ratio $(v)$, and mass density $(\rho)$. The total number of layers is generally unknown and specified/assumed by the analyst. The layer parameters are then varied by a search-algorithm until an acceptable match is made between the theoretical dispersion curve and the experimental dispersion data. However, the inverse problem involved in obtaining a realistic layered earth model from surface wave dispersion data is inherently ill-posed, nonlinear, and mix-determined, without a unique solution. The ill-posed nature of the problem results from trying to recover four parameters ( $\mathrm{t}, \mathrm{Vs}, \mathrm{Vp}$, and $\rho$ ) for each layer in the model indirectly from the two measured data parameters of Rayleigh phase velocity (Vr) and frequency (f). The problem is further complicated by the nonlinear relationship between the data parameters, which vary as a function of frequency/wavelength, and the desired model space parameters, which vary as a function of depth. Additionally, the model solution for deeper layers is dependent on the model solution for shallow layers, resulting in a mix-determined problem. As a result, a number of significantly 
different layered earth models may possess theoretical dispersion curves that fit the experimental data equally well (Foti et al. 2009 [16], Cox and Teague 2016 [15]).

Multiple studies have considered the variability in site response estimates derived from non-unique Vs profiles obtained from surface wave testing (e.g., Foti et al. 2009 [16], Boaga et al. 2011 [17], Jakka et al. 2014a [18], Griffiths et al. 2016b [19]). Conclusions drawn from these studies have been somewhat conflicting. For example, Foti et al. (2009) [16] used Monte Carlo inversions of synthetic and real datasets to conclude that Vs profiles with equivalent dispersion misfit values are essentially equivalent with regards to site response. On the other hand, Boaga et al. (2011) [17] argued that profiles with comparable dispersion misfit values may potentially exhibit significant variability with regards to site amplification if a sharp velocity contrast is not present beneath the site. Similarly, Jakka et al. (2014a) [18] argue that profiles with comparable misfit values exhibit significant variability with regards to site response. However, as pointed out by Comina and Foti (2014) [20], the theoretical dispersion curves associated with many of the Vs profiles in the Jakka et al. (2014a) [18] study fall outside of the uncertainty bounds of the experimental data at high frequencies, and do not follow the general trend/shape of the experimental data at low frequencies. Several follow up discussions have ensued between these differing schools of thought (e.g., Comina and Foti 2014 [20], Jakka et al. 2014b [21], Boaga et al. 2012 [22], Socco et al. 2012 [23]). One particular point of debate is focused on what constitutes "equivalence" in terms of dispersion misfit when attempting to select appropriate candidate Vs profiles for use in site response.

A companion study documented by Griffiths et al. (2016a and 2016b) [8], [19] investigated site response variability at two sites (one with a strong impedance contrast and one without) using many Vs profiles derived directly from a single surface wave inversion and from several statistically-based methods commonly used to account for epistemic uncertainty and aleatory variability. In the initial study, Griffiths et al. (2016a) [8] argued that the experimentally-measured dispersion data represents the "site signature", which reveals important information about wave propagation across the site, and any Vs profiles that fail to capture the site signature (i.e., fail to match the experimental dispersion data) may not be appropriate for trying to quantify the variability in site response. Inherent in this argument is the assumption that a broadband, high-quality experimental dispersion curve has been obtained and demonstrated to be representative of the site. The quality of fit in their study was quantified using the dispersion misfit equation of Wathelet et al. (2004) [24]:

$$
\text { misfit }=\sqrt{\frac{1}{n_{f}} \sum_{\mathrm{i}=1}^{\mathrm{n}_{\mathrm{f}}} \frac{\left(\mathrm{x}_{\mathrm{di}}-\mathrm{x}_{\mathrm{ci}}\right)^{2}}{\sigma_{\mathrm{i}}^{2}}}
$$

Where, $x_{d i}$ represents the Rayleigh wave phase velocity of the experimental dispersion data at frequency $f_{i} ; x_{c i}$ is the theoretical Rayleigh wave phase velocity computed for the trial layered earth model at frequency $f_{i} ; \sigma_{\mathrm{i}}$ is the standard deviation associated with the experimental dispersion data at frequency $f_{i}$; and $n_{f}$ is the number of frequency samples considered for the misfit calculation. The misfit value is essentially a root-mean-squared-error (RMSE) between the experimental and theoretical dispersion curves with a normalization factor equal to the inverse of the experimental standard deviation at a given frequency. According to this definition of misfit, a value less than 1.0 essentially means that on average (i.e., across the frequency band considered) the theoretical dispersion curve falls within the $+/$ - one standard deviation bounds of the 
experimental data. Thus, misfit values far in excess of 1.0 suggest a poor fit of the experimental dispersion data. Misfit values approaching zero represent better fits to the mean trend of the experimental data, and for a given set of data, dispersion misfit values can be used to make relative judgements regarding the quality of trial layered earth models. However, the authors acknowledge that misfit values deemed to be acceptable at one site may be considered unacceptable at another. For example, a misfit value of 0.9 might be "good" at one site because the theoretical dispersion curve visually fits a complicated dataset. However, a misfit value of 0.3 might be "bad" at another site because the theoretical dispersion curve does not agree well at all frequencies for a simple dispersion dataset. Thus, misfit values from different sites generally cannot be compared directly with one another for a measure of the overall inversion quality from site-to-site. Rather, the misfit values can simply be used to guide relative judgements about the quality of certain trial layered earth models relative to other potential models at the same site.

Griffiths et al (2016a) [8] found that theoretical dispersion curves associated with upper/lower range Vs profiles (e.g., median +/- 20\%) commonly used to account for epistemic uncertainty (e.g. Matasovic and Hashash 2012 [25], EPRI 2012 [7]) yielded a poor fit to the experimental dispersion data (i.e., relatively high misfit values) measured at their study sites. They also found that Vs randomization (Toro 1995 [26]) commonly utilized to account for aleatory variability resulted in only a few acceptable, and many unacceptable, Vs profiles based on dispersion misfit. However, Vs profiles derived directly from a surface wave inversion resulted in a satisfactory fit of the experimental dispersion data because the inversion algorithm seeks to achieve the best possible fit to the data and thus seeks profiles that best capture the experimentally-measured "site signature."

The follow-up study by Griffiths et al. (2016b) [19] documented linear and equivalent linear site response analyses performed using Vs profiles derived in their previous study. A total of $50 \mathrm{Vs}$ profiles with comparable misfit values derived from surface wave inversion were considered for each site. The response spectra (RS) and amplification factors (AF) associated with these profiles resulted in minimal variability, which supports the conclusions of Foti et al. (2009) [16]. On the other hand, the upper/lower range Vs profiles and the randomly-generated Vs profiles exhibited substantial variability in terms of RS and AF. The authors argue that since these statistically-generated profiles do not capture the "site signature", the variability that they exhibit may not be realistic, and may potentially result in underestimation of site response due to flattening-out of resonant frequencies. Overall, the results presented in Griffiths et al. (2016b) [19] show a strong, albeit not perfect, trend of increasing variability in equivalent linear site response estimates with increasing surface wave dispersion misfit values at their study sites.

It is important to briefly raise a few points regarding the experimental dispersion data obtained at real sites and the so-called "site signature". An experimental dispersion curve represents a spatial average of material properties over the length/area of the array used to measure surface waves (with the degree of averaging changing with frequency). Thus, if a single active-source survey (i.e., MASW or SASW) is conducted using an array that is relatively small in comparison to the area of interest, the resulting data cannot be deemed a "signature" of the site. Indeed, experimentally measured dispersion data measured using relatively small arrays at geologically-complex sites may vary considerably over short distances. For example, Thompson et al. (2012) [27] present dispersion data collected at two Kiknet sites in Japan, at which four independent spectral analysis of surface wave (SASW) surveys were conducted within a few hundred meters of one another. The experimental dispersion data at the first site was in excellent agreement over the frequency ranges that were resolved by the individual surveys. Thus, it can 
be argued that this site had a narrowly-defined site signature. Conversely, the experimental dispersion data derived from the four SASW surveys at the second site showed extreme variability. At such a geologically-complex site it may not be possible to establish a single dispersion curve with relatively narrow uncertainty bounds. Rather, the site signature may need to be characterized by a broad range of experimental dispersion data compiled from either a number of smaller arrays spread across the site and/or a combination of smaller active-source arrays and larger two-dimensional passive-source arrays that span the footprint of the site.

While many of the aforementioned studies examined the variability in site response estimates obtained using non-unique Vs profiles derived from surface wave inversion, each example considered only a single set of non-unique Vs profiles developed using the same set of inversion parameters (i.e., a single parameterization). In each case, the inversion algorithm was restricted to a single, predefined number of layers and ranges in their respective properties. As mentioned above, the inversion parameterization has been shown to significantly impact the results of a surface wave inversion (DiGiulio et al. 2012 [14], Cox and Teague 2016 [15]). This is because the number of unknowns, which is controlled by the number of layers in the parameterization, is itself an unknown. While Vs profiles developed from within a single set of parameters may exhibit significant differences, the differences between profiles derived from different sets of parameters has been shown to be even more significant. Indeed, it is possible to obtain comparable dispersion misfit values from profiles comprised of many layers with gradual increases in Vs and profiles with fewer layers and significant velocity contrasts. Without exploring different parameterizations comprised of different numbers of layers, it is not possible to fully capture the non-uniqueness associated with the inverse problem and the resulting variability in site response estimates.

Cox and Teague (2016) [15] outlined a systematic procedure to investigate potential inversion parameterizations, each with a unique number of layers defined by a specific layering ratio. This procedure aids in avoiding blatantly under- and over-constrained parameterizations. However, even when this procedure is used, it is possible to have multiple inversion parameterizations that are adequately constrained, yet produce significantly different Vs profiles. In such cases, it may be difficult to decide which Vs profiles best represent the "true" subsurface layering without a-priori information, and the overall influence of this non-uniqueness on site response is of interest.

The present study expands on the results presented in Cox and Teague (2016) [15]. Specifically, linear-elastic and equivalent-linear site response analyses using both low- and highintensity input ground motions have been performed on Vs profiles from the semi-synthetic blind-study site inverted in Cox and Teague (2016) [15]. Vs profiles considered include those that were developed from under-, over-, and adequately-constrained inversion parameterizations. Additionally, commonly-used strategies of accounting for Vs profile uncertainty and its influence on the predicted site response are considered. These commonly-used strategies of accounting for Vs uncertainty include upper/lower range base-case Vs profiles meant to account for epistemic uncertainty and statistically-based, randomly generated Vs profiles meant to account for aleatory variability.

With regards to surface wave testing, epistemic uncertainty represents data uncertainty and/or lack of scientific knowledge regarding mode interpretations and which processing and inversion algorithms are "best", while aleatory variability reflects the vertical and horizontal spatial variability of Vs across the site, which also contributes to data uncertainty. While the uncertainty associated with the inversion itself is purely epistemic, the data used as input into the 
inversion (i.e., the experimental dispersion data) is influenced by both aleatory variability and epistemic uncertainty. Therefore, the final Vs profiles derived directly from surface wave testing are influenced by both sources of uncertainty and it would be very difficult to decouple them (Griffiths et al. 2016a [8]). In this study, no attempt was made to do so. Consequently, any uncertainty/variability in site response performed on these Vs profiles also reflects both sources of uncertainty/variability. However, we do not claim that the surface wave dispersion approach of accounting for Vs uncertainty will encompass all sources/forms of aleatory variability and epistemic uncertainty associated with non-unique Vs profiles used in site response. For example, if a surface wave survey is conducted over an area that is relatively small compared to the area of interest, then it cannot capture the full aleatory variability. Moreover, if an experimental dispersion curve does not extend to low enough frequencies to constrain the depth to bedrock, then the epistemic uncertainty regarding the depth to bedrock cannot be reduced and multiple base-case Vs profiles accounting for this uncertainty may be required. Nonetheless, if high quality surface wave surveys are conducted over the area of interest, it may be possible to substantially reduce site response uncertainty by only considering Vs profiles that realistically fit the site signature.

\section{$\underline{\text { InterPACIFIC Blind-Study Site } 4}$}

The dataset considered in this paper was originally provided to participants in the InterPACIFIC (Intercomparison of methods for site parameter and velocity profile characterization) project. While Garafalo et al. (2016a) [9] and (2016b) [10] document the results obtained from three InterPACIFIC blind-study sites in Europe, the results from blind-study Site 4 have not yet been published. Thus, we refer only to our own results and methodologies herein.

The experimental data for Site 4 was provided by Cecile Cornou, from ISTerre (Institut des Sciences de la Terre), Grenoble, France. The semi-synthetic experimental data was developed by first performing a surface wave inversion on experimentally-measured Rayleigh wave dispersion data from a real site. The minimum misfit ground model from this analysis was then chosen as the "true" solution profile for Site 4. The theoretical dispersion curve for this true solution profile was discretized at the frequencies corresponding to the experimentally-measured dispersion data from the real site. Additionally, the frequency-dependent uncertainty bounds associated with the original experimentally-measured dispersion data were applied to this discretized theoretical dispersion curve. This produced a pseudo-experimental dispersion curve, with the mean value at each frequency corresponding to the theoretical Rayleigh phase velocity of the true solution Vs profile and the uncertainty bounds reflecting realistic uncertainty from actual surface wave testing. Although this curve was not measured directly, it will be referred to as the "experimental dispersion curve" for the remainder of the paper.

All participants in the project were provided with the experimental dispersion curve for Site 4 and asked to invert the data in a blind manner (i.e., no a-priori information about the site was made available). The mean experimental dispersion data provided to the blind-study participants, including +/- one standard deviation bounds, are shown in Figure 1a. Analysts were informed that the data represented the fundamental Rayleigh mode, eliminating the need to consider the possibility of effective or higher modes in their inversions. Each analyst was asked to submit a single "best" Vs profile, and were allowed to also submit a range of Vs profiles that accounted for Vs uncertainty if they so desired. After final submissions, a mean and $+/$ - one standard deviation horizontal-to-vertical (H/V) spectral ratio curve (i.e. the ratio between the Fourier amplitude spectra of the horizontal and vertical components of ambient vibrations) 
measured at the real site and the true solution Vs profile for Site 4 were made known to the participants. The H/V curves and true solution Vs profile are shown in Figure 1b and Figure 1c, respectively. The true solution Vs profile is defined by a large velocity contrast at $56 \mathrm{~m}$, which is the cause of the sharp peak in the H/V curve at $1.6 \mathrm{~Hz}$.
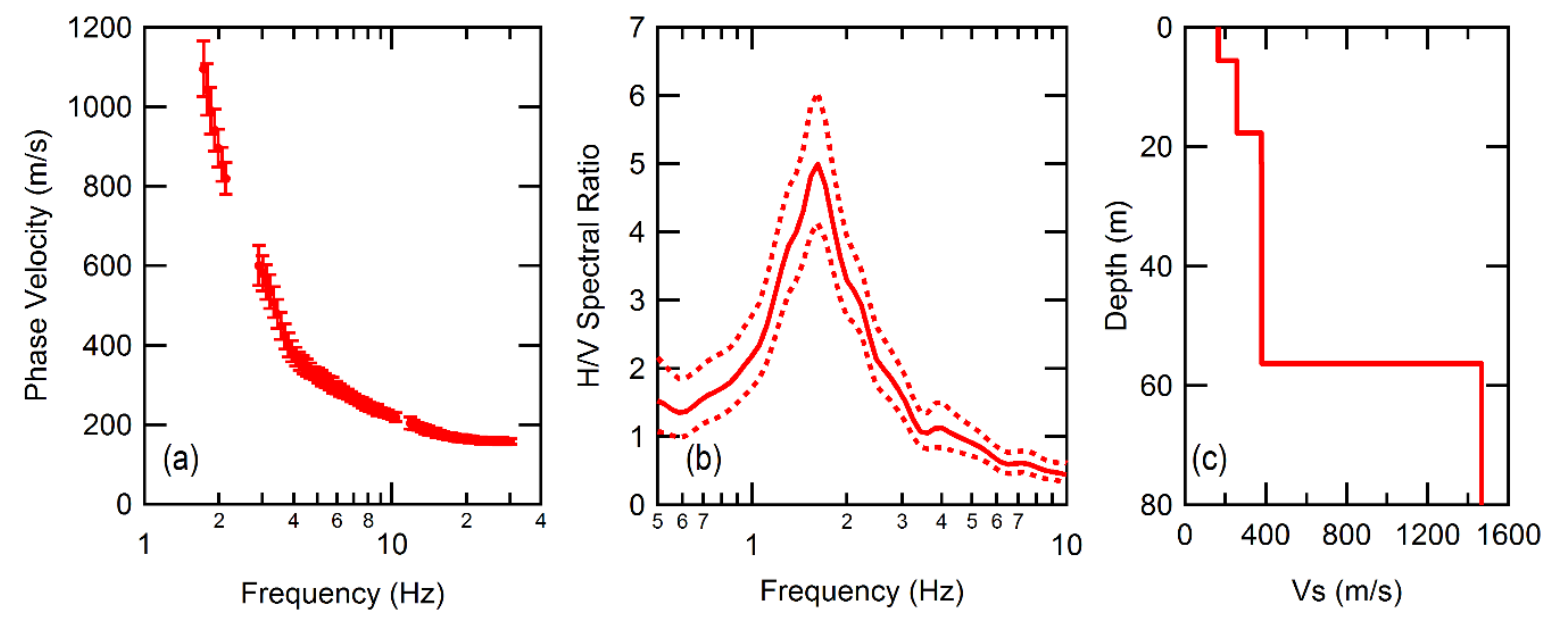

Figure 1: InterPacific blind-study Site 4: (a) dispersion data, (b) mean (solid line) and +/- one standard deviation (dotted lines) H/V spectral ratio curves, and (c) true solution Vs profile.

\section{Vs Profiles used in Site Response}

Vs profiles considered in this study include: (1) $350 \mathrm{Vs}$ profiles developed by performing multiple inversions, each with a unique set of layering parameters, on the blind-study Site 4 dispersion data shown in Figure 1a; (2) two upper/lower range Vs profiles developed by systematically increasing or decreasing the Site 4 true solution Vs profile by 20\%; and (3) 100 profiles developed using the Vs randomization procedure proposed by Toro (1995) [26]. Before discussing the results of the site response analyses, it is important to provide background information on how the Vs profiles used in this study were developed. This discussion will serve as a basis for considering which Vs profiles can be considered useful for quantifying the variability in site response estimates.

\section{Vs Profiles Derived from the Layering Ratio Surface Wave Inversion Technique}

After receiving the experimental dispersion data for blind-study Site 4, we performed multiple inversions on the data using the layering ratio approach detailed in Cox and Teague (2016) [15] and described briefly in the following paragraphs. Inversions were performed using the Geopsy software (www.geopsy.org). Geopsy utilizes a neighbourhood algorithm and the misfit function detailed in Equation 1 to search for the "best" layered earth models within a predefined inversion parameter space (Wathelet et al. 2004 [24]). The theoretical dispersion forward computations for each trial earth model are based on the work originally developed by Thomson (1950) [28] and Haskell (1953) [29] and later modified by Dunkin (1965) [30] and Knopoff (1964) [31]. 
Seven distinct inversions were performed on the experimental dispersion data from Site 4. Each inversion utilized a unique set of parameters, where the number of trial layers was defined by a unique layering ratio $(\Xi)$. The layering ratio represents a multiplier that systemically increases the range of possible depths to the bottom of each layer in the parameterization based on the range of possible depths to the bottom of the layer directly above it. By considering multiple layering ratios, it is possible to systematically investigate non-unique Vs profiles that could be adversely influenced by either too many or too few layers. Small layering ratios yield many thin trial layers in an inversion, while large layering ratios yield only a few thick trial layers in an inversion. Initially, six inversions were performed using layering ratios of 1.2, 1.5, 2.0, 3.0, 3.5 and 5.0. Subsequently, the inversion parameters corresponding to a layering ratio of 3.5 were deemed to be most representative of subsurface conditions and were slightly modified using engineering judgement in order to achieve an even better fit of the experimental data. This inversion is referred to as $3.5^{*}$. Approximately 200,000 layered earth models were explored during each layering ratio inversion. An ensemble of the 1,000 lowest misfit profiles was selected to represent each analysis. Of these 1,000 Vs profiles, 50 were randomly selected for use in subsequent site response analyses. The number 50 was chosen because it is manageable, from a computational standpoint, and because $50 \mathrm{Vs}$ profiles chosen randomly from the population of the best $1,000 \mathrm{Vs}$ profiles was found to statistically reproduce the same median and standard deviation as the population of 1,000. The same conclusions were reached at the two sites discussed in Griffiths et al. (2016a) [8]. Thus, 350 total Vs profiles resulting from surface wave inversion were selected for site response (i.e., $50 \mathrm{Vs}$ profiles from 7 unique inversions of the same dataset).

The 50 Vs profiles selected from each layering ratio inversion are shown along with their corresponding theoretical dispersion curves relative to the experimental dispersion data in Figure 2. The range of misfit values for the 50 theoretical dispersion curves associated with each layering ratio inversion are shown in brackets within the dispersion curve subfigures (i.e., Figures $2 \mathrm{a}, 2 \mathrm{c}, 2 \mathrm{e}, 2 \mathrm{~g}, 2 \mathrm{i}$, and 2k). Upon inspection, it is clear that the theoretical dispersion curves visually fit the experimental data extremely well for all layering ratios, making it difficult to distinguish individual curves. Furthermore, the maximum misfit values for each inversion are all less than 0.5 , and closer to 0.25 or less on average. Thus, if only a single one of these inversions had been performed for the site, an analyst may have been inclined to believe that the resulting Vs profiles were a reasonable representation of the subsurface. However, upon inspection of the $50 \mathrm{Vs}$ profiles obtained from each layering ratio inversion (i.e., Figures $2 \mathrm{~b}, 2 \mathrm{~d}$, $2 \mathrm{f}, 2 \mathrm{~h}, 2 \mathrm{j}$, and $2 \mathrm{l}$ ), one can clearly see that many of the Vs profiles do not well-represent the true solution for the subsurface stiffness profile. The closest representations were obtained using layering ratios of 3.5 and $3.5^{*}$ (Figure $2 \mathrm{j}$ ), and the dispersion misfit values associated with these layering ratios (Figure 2i) are in some cases an order of magnitude lower than those associated with higher or lower layering ratios. In the original InterPACIFIC project blind study, the profiles associated with a layering ratio of $3.5^{*}$ were deemed by the authors to be the "best" representation of the subsurface (keeping in mind that we did not have the true solution profile at the time) and the median Vs profile of this ensemble was submitted as our single best Vs profile. However, for a real site, where the true answer is unknown, and in the typical case of sparse borehole data horizontally and vertically, one may struggle to know what the "best" answer is and how to realistically account for Vs uncertainty. Thus, these results underscore some of the challenges associated with the non-unique nature of surface wave inversion. For example, Vs profiles associated with a layering ratio of 1.2 (i.e., many thin layers) show gradual increases in 
Vs with depth, while Vs profiles associated with a layering ratio of 5.0 (i.e., fewer thick layers) exhibit significant velocity contrasts at the wrong depths. These strong velocity contrasts (or lack thereof) are of particular interest in site response analyses. Moreover, they also play a significant role in probabilistic seismic hazard analyses (PSHA) because many ground motion prediction equations incorporate velocity horizons (e.g. depth to 1.0 and $2.5 \mathrm{~km} / \mathrm{s}$ shear-wave velocity horizons, or $Z_{1.0}$ and $Z_{2.5}$, respectively). Thus, these velocity contrasts also influence the selection of input ground motions prior to the site response analyses.

Interestingly, Cox and Teague (2016) [15] argued that they would have discarded the results from layering ratios of 1.2 and 5.0 as not likely representative of the true subsurface profile, even without a-priori information about the site layering. This argument was based on consideration of both the dispersion misfit values and the extreme variability (or lack thereof) in the best 1,000 Vs profiles. For example, the relatively high misfit values associated with a layering ratio of 5.0 (in comparison to those achieved with a layering ratio of 3.5), the narrow range of misfit values, and the minimal variability of the Vs profiles suggest that the inversion parameterization is overly-restrictive and incorporates too few layers. Essentially, the inversion algorithm found, and settled for, the best possible solution within an overly-restrictive parameterization. Additionally, they claim that the parameters associated with a layering ratio of 1.2 are too permissive. This claim is made based on the fact that significantly better fits of the experimental dispersion data were achieved using far fewer layers (i.e., higher layering ratios) given the same number of searched trial models. Moreover, the high variability in the Vs profiles using a layering ratio of 1.2 suggests that the inversion algorithm may not have been able to find the most promising regions of such a broad parameter space and/or that too many degrees of freedom result in highly variable solutions. Thus, in some of the discussions presented below, Vs profiles derived from layering ratios of 1.2 and 5.0 will not be included when trying to realistically quantify Vs uncertainty at the site. These cases will be clearly noted. Nonetheless, in practice, it can be difficult to conclude if a parameterization is too restrictive or too broad without significant experience and/or a wealth of other subsurface data. Thus, Vs profiles from layering ratios of 1.2 and 5.0 will still be included in subsequent site response analyses as a means to determine the impact of unintentionally using poorly-parametrized models.

In order to conduct site response analyses, all profiles shown in Figure 2 were truncated at the first layer with a Vs exceeding $1,000 \mathrm{~m} / \mathrm{s}$. This layer, whatever the absolute velocity was, so long as it exceeded $1,000 \mathrm{~m} / \mathrm{s}$, was chosen as the half-space layer for input of ground motions (GMs). A cut-off value of $1,000 \mathrm{~m} / \mathrm{s}$ whose chosen, somewhat arbitrarily but after thoughtful consideration, because it was within the bounds of NHERP Site Class B rock site conditions (i.e., $760-1500 \mathrm{~m} / \mathrm{s}$ ), and it would yield Vs profiles with a range of half-space velocities that encompassed the velocity of the half-space in the solution profile (i.e., about $1,500 \mathrm{~m} / \mathrm{s}$ ). As a result, half-space velocities for the $350 \mathrm{Vs}$ profiles determined via surface wave inversion ranged from just over $1,000 \mathrm{~m} / \mathrm{s}$ to greater than $3,000 \mathrm{~m} / \mathrm{s}$. Furthermore, the depths for the half-space layers ranged from less than $50 \mathrm{~m}$ to over $150 \mathrm{~m}$. 

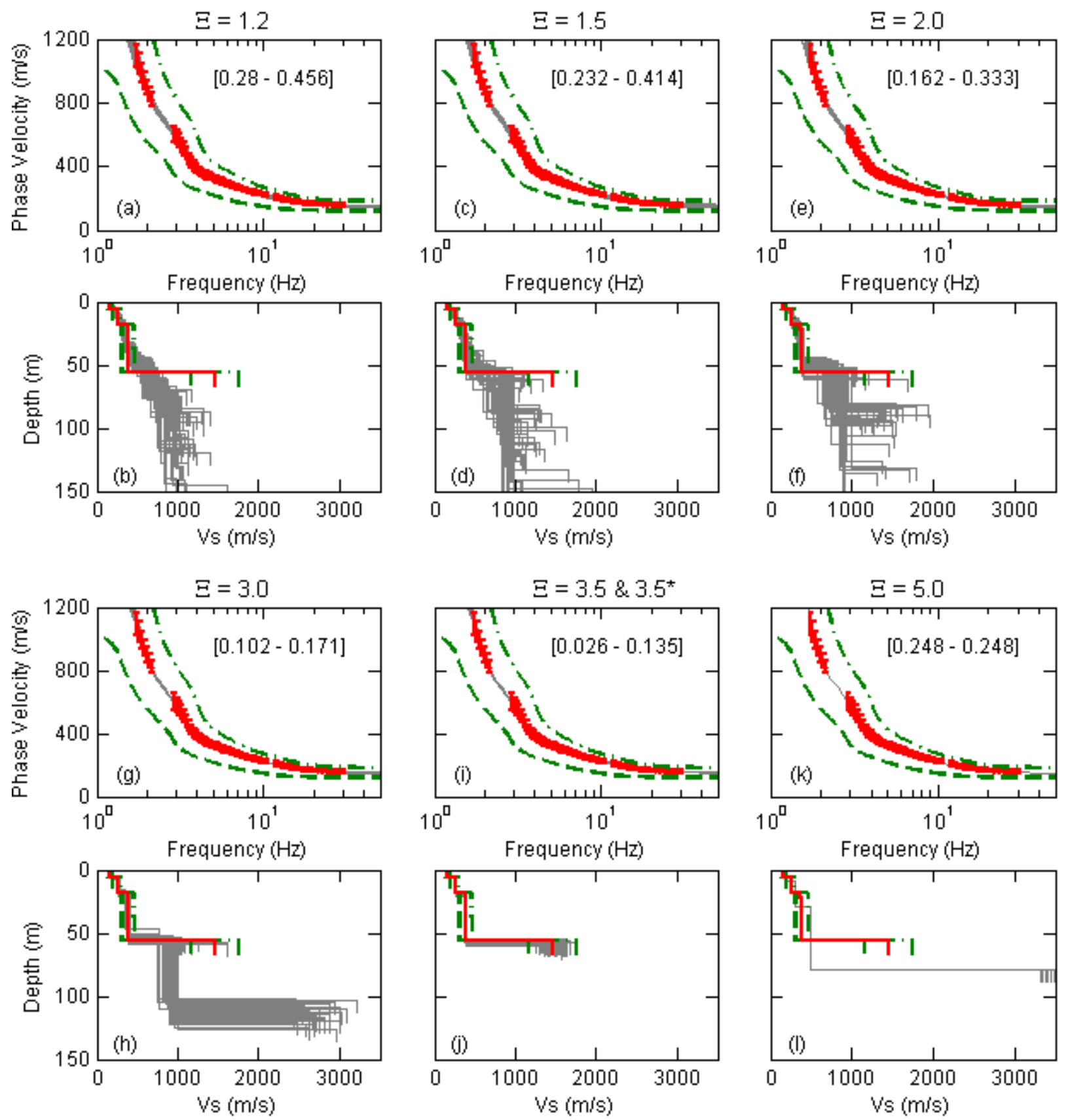

$$
\begin{gathered}
\hline \text { Solution }--- \text { Solution - 20\% [8.41] - '- - Solution + 20\% [3.76] } \\
\hline \text { Inversion } \quad \text { Experimental Dispersion Curve }
\end{gathered}
$$

Figure 2: Fifty theoretical dispersion curves and 50 corresponding Vs profiles, respectively, obtained from surface wave inversions based on the following layering ratios: (a, b) 1.2, (c, d) 1.5, (e, f) 2.0, (g, h) 3.0, (i, j) 3.5 and 3.5*, and $(\mathrm{k}, 1)$ 5.0. Note that the numbers in brackets represent dispersion misfit values. Each set of $50 \mathrm{Vs}$ profiles were randomly sampled from a suite of the 1,000 lowest misfit profiles obtained from each layering ratio inversion. Also shown are the theoretical dispersion curves and the Vs profiles corresponding to the solution profile $+/-$ $20 \%$. 


\section{Upper/Lower Range and Statistically-Based, Randomly Generated Vs Profiles}

In addition to performing site response analyses on Vs profiles derived directly from a surface wave inversion, additional techniques of accounting for Vs uncertainty were considered. These techniques included the use of upper/lower range profiles to account for epistemic uncertainty and the use of statistically-based, randomly generated Vs profiles to account for aleatory variability. The development of these Vs profiles is discussed below.

For this study, the true solution was chosen as the "mean" base-case Vs profile. The "mean" base-case Vs profile was then increased and decreased by $20 \%$ to develop upper/lower range base-case profiles for use in site-response analyses. The upper/lower range profiles are shown relative to the solution Vs profile and the inversion Vs profiles in Figure 2. These profiles look very reasonable when considering the variability among the Vs profiles derived from the various surface wave inversion parameterizations. In fact, one could argue that they significantly underestimate the uncertainty in Vs resulting from surface wave inversion. However, as discussed in Griffiths et al. (2016a) [8], it is useful to compute theoretical dispersion curves for these Vs profiles and assess whether or not they match the experimental dispersion data. As shown in Figure 2, theoretical dispersion curves for the $+/-20 \%$ profiles fall well above and well below the experimental dispersion data, with misfit values for the $+/-20 \%$ profiles of 3.76 and 8.41 , respectively. Thus, while the upper/lower range Vs profiles visually appear to be a much better representation of the true solution Vs profile than many of the profiles derived from surface wave inversion, their theoretical dispersion curves do not fit the experimental data nearly as well. It should be noted that in order to compute theoretical dispersion curves for these upper/lower range profiles, assumptions were made regarding $\mathrm{Vp}$ and mass density. In order to be consistent, we used the same assumptions utilized during inversion of the surface wave data. Namely, Vp was constrained using reasonable values for Poisson's ratio above the water table (i.e., $0.25-0.33$ ) and by assuming a value of $1,500 \mathrm{~m} / \mathrm{s}$ below the water table, unless Vs exceeded $750 \mathrm{~m} / \mathrm{s}$, at which point Poisson's ratio was again utilized to estimate Vp. While, the mass density has minimal influence on the theoretical dispersion curve (Wathelet 2004 [24]), reasonable ranges for geomaterials of various stiffness are well-established.

The Vs randomization model proposed by Toro (1995) [26] was used in this study to investigate common methods of accounting for aleatory variability. Once again, the true solution was chosen as the baseline Vs profile about which Vs randomization would occur. The Toro (1995) [26] model has been implemented in the software STRATA (Kottke and Rathje 2009 [32]) and operates on the following three categories of parameters: (1) the Vs statistical parameters, (2) the layering parameters, and (3) the depth to bedrock parameters. Two distinct sets of randomized Vs profiles were developed using the Toro model in order to investigate the impact of using different sets of model parameters. Where possible, site-specific parameters were used to develop the first set of randomized Vs profiles, while default/recommended parameters corresponding to sites with a $\mathrm{V}_{\mathrm{S} 30}$ ranging from 180 to $360 \mathrm{~m} / \mathrm{s}$ (USGS C in Table 5 of Toro (1995) [26]) were used to develop the second set. These randomized Vs profiles are referred to for the remainder of the paper as the "site-specific Toro profiles" and the "default Toro profiles", respectively. The parameters used to generate each set are discussed below and summarized in Table 1. 
In order to compute site-specific Toro model parameters, a population of $250 \mathrm{Vs}$ profiles was created by combining each of the sets of $50 \mathrm{Vs}$ profiles developed from surface wave inversion using layering ratios of $1.5,2.0,3.0,3.5$ and $3.5^{*}$. As noted above, the Vs profiles derived from layering ratios of 1.2 and 5.0 were not used to develop site-specific Toro parameters because they were obtained from inversions judged to be poorly-parameterized by Cox and Teague (2016) [15]. The population of $250 \mathrm{Vs}$ profiles is shown in Figure 3a relative to the solution profile. As noted earlier, both aleatory variability and epistemic uncertainty are inherent in this population of Vs profiles. However, the Toro (1995) [26] model is theoretically used solely to account for aleatory variability (EPRI 2012) [7]. Thus, one could argue that this approach improperly mixes the two types of uncertainty. However, there is certainly epistemic uncertainty associated with the Vs profiles used to develop the Toro (1995) [26] model because epistemic uncertainty is inherent in all techniques used to measure Vs. For example, wave travel paths are often assumed to be straight lines between the source and receiver in crosshole and downhole seismic testing, yet in reality the travel paths and arrival time picks are uncertain and may be quite complex. This underscores the difficulty in perfectly decoupling aleatory variability and epistemic uncertainty. In any case, we acknowledge that in an effort to decouple some of the epistemic uncertainty, a representative base-case Vs profile could be developed for each unique layering ratio, with randomization parameters developed using only Vs profiles from that single layering ratio. This approach may be warranted and requires further study. However, given the large number of base-cases, this approach may not be palatable to many practitioners.

The depth to bedrock in the solution profile, which corresponds to the base-case $\left(\mathrm{z}_{\text {rock }}\right)$, is indicated by a Vs jump from approximately 400 to $1,500 \mathrm{~m} / \mathrm{s}$ at a depth of $56 \mathrm{~m}$. In order to quantify the uncertainty associated with this depth, as required by the Toro (1995) [26] model, the variable depths to bedrock associated with the Vs profiles derived from surface wave inversion were used. As mentioned above, the depth to bedrock for each Vs profile derived from surface wave inversion was determined to coincide with the first layer to exceed $1,000 \mathrm{~m} / \mathrm{s}$. These depths to bedrock were weighted by the inverse of their dispersion misfit values (i.e. the inverse of Eq. 1) prior to calculating the lognormal standard deviation $\left(\sigma_{\text {lnZrock }}\right)$. Meaning, Vs profiles with lower dispersion misfit values were given higher weights since they were believed to more accurately represent subsurface conditions. Note that the lognormal distribution does not perfectly match the depth to bedrock data. However, the Toro (1995) model as implemented in STRATA requires that the depth to rock be modelled with either a uniform, normal, or lognormal distribution (Kottke and Rathje 2009 [32]) and the lognormal distribution was found to be most representative with $\sigma_{\text {lnZrock }}=0.33$. The $+/$ - one standard deviation values for the depth to bedrock are shown relative to the Vs profiles in Figure $3 \mathrm{a}$. Note that there is no default/recommended depth to rock or standard deviation for depth to rock in the Toro (1995) model. Thus, these site-specific values were also used to generate the default Toro Vs profiles discussed below (refer to Table 1).

Vs statistical parameters for the Toro (1995) [26] model include the depth-dependent lognormal standard deviation of $\mathrm{Vs}\left(\sigma_{\operatorname{lnV} s}\right)$ and the inter-layer correlation parameters $\left(\Delta, \mathrm{d}_{0}, \mathrm{~b}, \rho_{0}\right.$, and $\left.\rho_{200}\right)$. $\sigma_{\operatorname{lnVs}}$ versus depth was computed for the population of $250 \mathrm{Vs}$ profiles and is shown in Figure 3 b. Once again, for the calculation of $\sigma_{\ln V s}$, each Vs profile was weighted by the inverse of its misfit value, resulting in more weight being applied to those profiles whose dispersion data 
best matches the experimental dispersion data. $\sigma_{\operatorname{lnVs}}$ ranges from 0.04 near the ground surface to 0.22 below approximately $60 \mathrm{~m}$. It is clear that there is significantly more uncertainty involved in estimating the depth/velocity of bedrock from surface wave inversions than in estimating soil velocities. This finding has been noted by others (e.g., Cornou et al. 2009 [12], Garafalo et al. 2016a [9]). A robust determination of site-specific Vs inter-layer correlation parameters $\left(\Delta, \mathrm{d}_{0}, \mathrm{~b}\right.$, $\rho_{0}$, and $\rho_{200}$ ) is beyond the scope of this study. Thus, recommended values were used in both the default and site-specific analyses (see Table 1).

Regarding the "default" $\sigma_{\operatorname{lnVs}}$ values, it should be noted that Toro (1995) [26] provides both "generic" $\sigma_{\operatorname{lnVs}}$ based on seismic site class and "site-specific" $\sigma_{\operatorname{lnVs}}$ computed from "clusters" of Vs profiles (not to be confused with the site-specific $\sigma_{\operatorname{lnVs}}$ for Site 4 based on Vs profiles from surface wave inversion, as discussed in the previous paragraph). Based on the most reliable "clusters" (i.e., those containing more than $10 \mathrm{Vs}$ profiles), a site-specific $\sigma_{\ln V s}$ of 0.15 between 0 and $50 \mathrm{~m}$ and 0.22 at greater depths is recommended in Stewart et al. (2014) [33]. The "generic" $\sigma_{\ln V s}$ for sites with a $\mathrm{V}_{\mathrm{S} 30}$ ranging from 180 to $360 \mathrm{~m} / \mathrm{s}$ is 0.31 , which is significantly higher than the site-specific values. In practice, the site-specific $\sigma_{\ln V s}$ should be used when a sitespecific Vs profile is available and geologic variability across the site is modest (Stewart et al. 2014 [33]). For this study, randomized Vs profiles were developed using both the site-specific values recommended in Stewart et al. (2014) [33] and the generic $\sigma_{\ln V s}$ values, however, only the results for the generic $\sigma_{\operatorname{lnV}}$ are shown here. Although this is not the optimum approach when a site-specific Vs profile is available, we chose to show the results associated with the generic $\sigma_{\ln V s}$ for two reasons. First, the Vs profiles developed using the site-specific $\sigma_{\operatorname{lnVs}}$ from Stewart et al. (2014) [33] yielded results that were fairly similar to those obtained using the site-specific parameters that we developed using the inversion Vs profiles from Site 4. Indeed, the $\sigma_{\operatorname{lnVs}}$ in Stewart et al. (2014) [33] and the $\sigma_{\mathrm{lnVs}}$ computed from the inversion Vs profiles at Site 4 are equal to one another below a depth of $60 \mathrm{~m}$. Moreover, the generic $\sigma_{\ln V s}$ values are programed in STRATA, represent a more extreme case, and are commonly used.

The Toro (1995) [26] model develops the layering for a Vs profile using a nonhomogeneous Poisson process where the occurrence rate $\left(\lambda_{t}\right)$ is a function of depth. The occurrence rate defines the distance between layer boundaries (i.e., thickness) and has units of the inverse of distance. The number of expected layer interfaces over a given depth interval decreases with occurrence rate (i.e., layer thickness generally increases with decreasing occurrence rate). Thus, occurrence rate is generally higher near the ground surface where thinner layers may be resolved, and lower at depth where layers are assumed to be thicker and the ability to resolve thin layers diminishes with most invasive and non-invasive methods. The Toro model uses three parameters, referred to as a, b and c in STRATA [32] (or $\mathrm{c}_{3}, \mathrm{c}_{1}$, and $-\mathrm{c}_{2}$ in Toro (1995) [26], respectively), to define the occurrence rate. A site-specific occurrence rate was developed by plotting the inverse of layer thickness versus mid-depth for all layers associated with the 250 Vs profiles in the population. The parameters $a, b$, and c were varied until a visually-satisfactory fit to the data was achieved. The site specific and default parameters are provided in Table 1, and the occurrence rates as a function of depth are plotted in Figure $3 \mathrm{c}$. Note that the default occurrence rate is lower than the site-specific relationship near the ground surface, resulting in thicker near-surface layers for the default case. However, the default occurrence rate is higher 
than the site-specific relationship over most depths, resulting in thinner layers for the default case at depth.

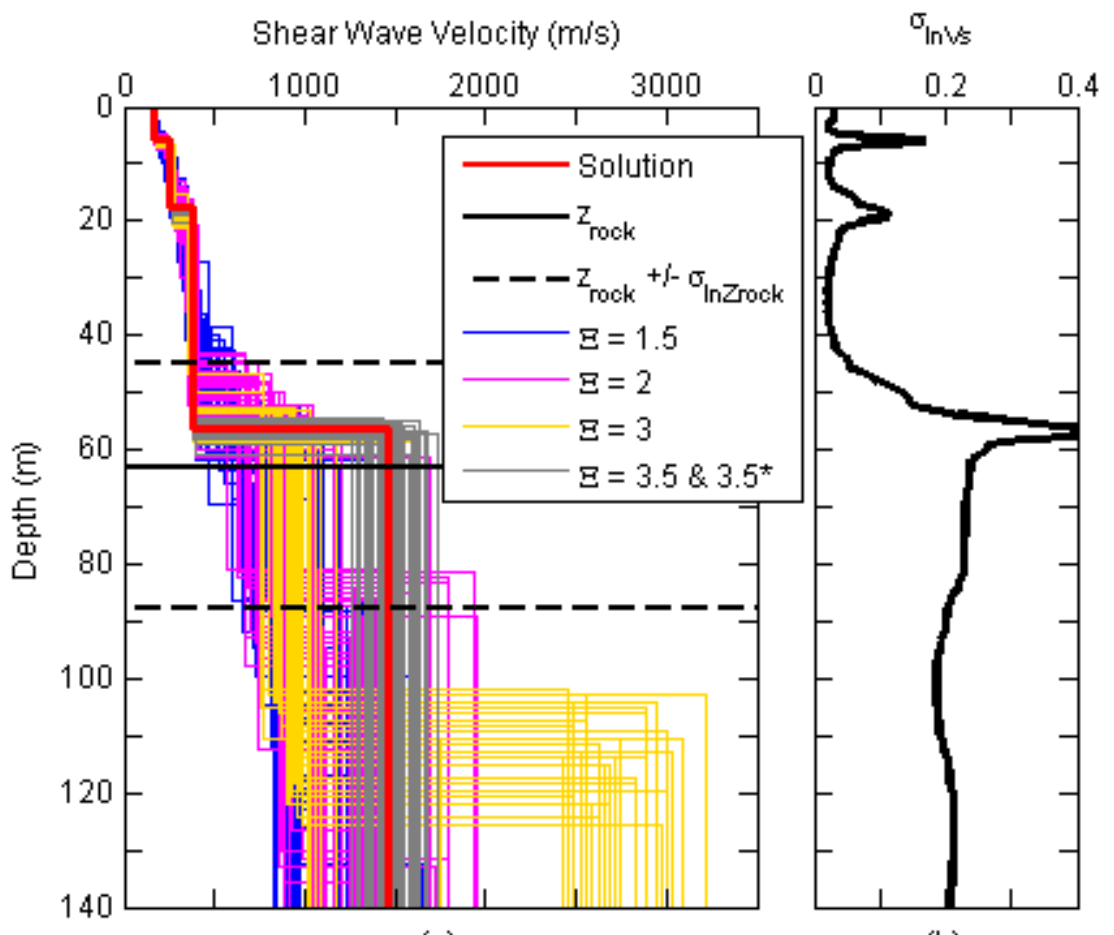

(a)

(b)
Occurrence Rate $\left(\mathrm{m}^{-1}\right)$

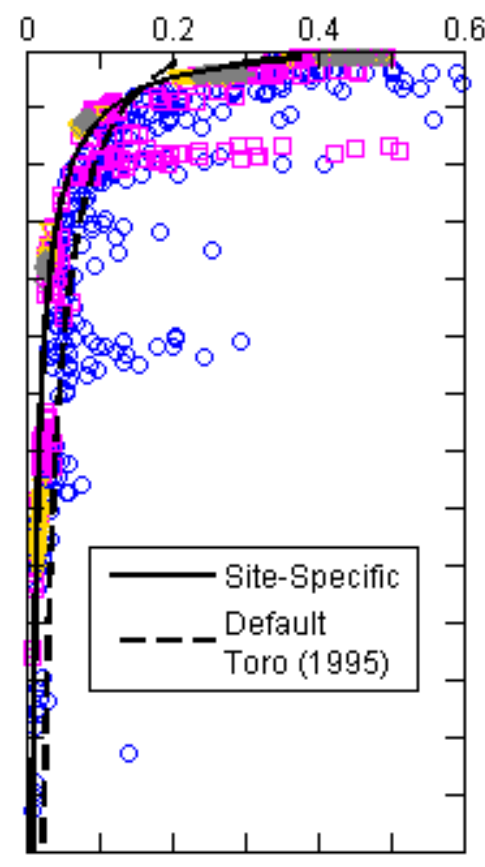

(c)

Figure 3: (a) Population of $250 \mathrm{Vs}$ profiles derived from layering ratio surface wave inversions used to develop site-specific Toro (1995) randomization parameters. Site-specific parameters used in Toro randomization include: (a) standard deviation of the natural logarithm of the depth to bedrock, (b) standard deviation of natural logarithm of Vs as a function of depth, and (c) transition rate relationship.

Table 1: Default and site-specific Toro (1995) randomization parameters used in this study

\begin{tabular}{|c|c|c|c|c|c|c|c|}
\hline \multicolumn{3}{|c|}{ Vs statistical parameters } & \multicolumn{2}{|c|}{ Layering parameters } & \multicolumn{3}{|c|}{ Depth to bedrock parameters } \\
\hline & Default $^{\mathrm{A}}$ & Site-specific & Default & Site-specific & & Default $^{\mathrm{B}}$ & Site-s pecific \\
\hline$\sigma_{\operatorname{lnVs}}$ & 0.31 & 0.04 to 0.22 & 1.98 & 2.4 & base-case $[\mathrm{m}]$ & 56 & 56 \\
\hline$\Delta$ & 3.9 & default & 10.86 & 4.0 & $\sigma_{\text {lnZrock }}$ & 0.33 & 0.33 \\
\hline $\mathrm{d}_{0}$ & 0 & default & -0.89 & -1.15 & & & \\
\hline b & 0.344 & default & & & & & \\
\hline$\rho_{0}$ & 0.99 & default & & & & & \\
\hline$\partial_{200}$ & 0.98 & default & & & & & \\
\hline
\end{tabular}

A. Default for sites with a $\mathrm{V}_{\mathrm{S} 30}$ ranging from 180 to $360 \mathrm{~m} / \mathrm{s}$

B. No default/recommended values provided in Toro (1995). Site-specific values were used.

The site-specific and default Toro model parameters were used to develop 50 random site-specific Vs profiles and 50 random default Toro Vs profiles, respectively, as shown in Figure $4 \mathrm{a}$ and $4 \mathrm{c}$. The surficial layers are generally thinner and less variable for the site-specific 
Toro profiles, which is due to the higher occurrence rate and significantly lower $\sigma_{\ln V s}$ values used to constrain the near-surface velocities (refer to Table 1). The default Toro profiles exhibit significantly more variability at all depths, which stems primarily from the relatively high default $\sigma_{\text {lnVs. }}$ The depth to rock in the randomized Vs profiles is quite variable in both cases, stemming from the relatively large standard deviation on the depth to bedrock that was used in the model $\left(\sigma_{\text {lnZrock }}=0.33\right)$. However, generally speaking, the median Vs profile derived from each set of 50 Toro Vs profiles matches the solution/mean base-case profile relatively well down to a depth of about $56 \mathrm{~m}$, which corresponds to the depth to bedrock. Thus, while the individual Vs profiles developed from randomization can be highly erratic, on average they tend to recover the basecase profile relatively well, particularly for the site-specific Toro profiles.

Theoretical dispersion curves were computed for each randomized Vs profile developed using the Toro (1995) [26] model. Again, consistent and reasonable assumptions were made regarding $\mathrm{Vp}$ and mass density for these calculations. Theoretical dispersion curves for the sitespecific and default Toro profiles are shown in Figure $4 \mathrm{~b}$ and $4 \mathrm{~d}$, respectively. Dispersion misfit values are shown in brackets within the figures. These misfits are generally one to two orders of magnitude greater than those associated with the profiles developed using the layering ratio surface wave inversion approach (Figure 2), which will be collectively referred to as the "inversion profiles" for the remainder of the paper. Most theoretical dispersion curves associated with both sets of Toro profiles would not be deemed acceptable by even the most permissive of standards, as they lie well above/below the uncertainty bounds of the experimental data. This is especially true for the dispersion curves associated with the default Toro Vs profiles, many of which are well outside the dispersion curves associated with the $+/-20 \%$ profiles. Since the Toro profiles generally fall well above or below the experimental dispersion data at all frequencies, the poor misfit values cannot be attributed to a single factor such as the depth to bedrock. This poor representation of the experimental dispersion data by both the upper/lower range base-case and randomized Vs profiles is worth careful consideration when attempting to realistically quantify Vs uncertainty for site response. If the experimental dispersion data is demonstrated to be relatively constant across a site, it could be argued that Vs profiles that do not fit the data are over-estimating the aleatory variability. However, if surface wave testing has only been performed over a relatively small footprint, then this poor representation of the experimental dispersion data may be deemed acceptable, but only after careful consideration. 

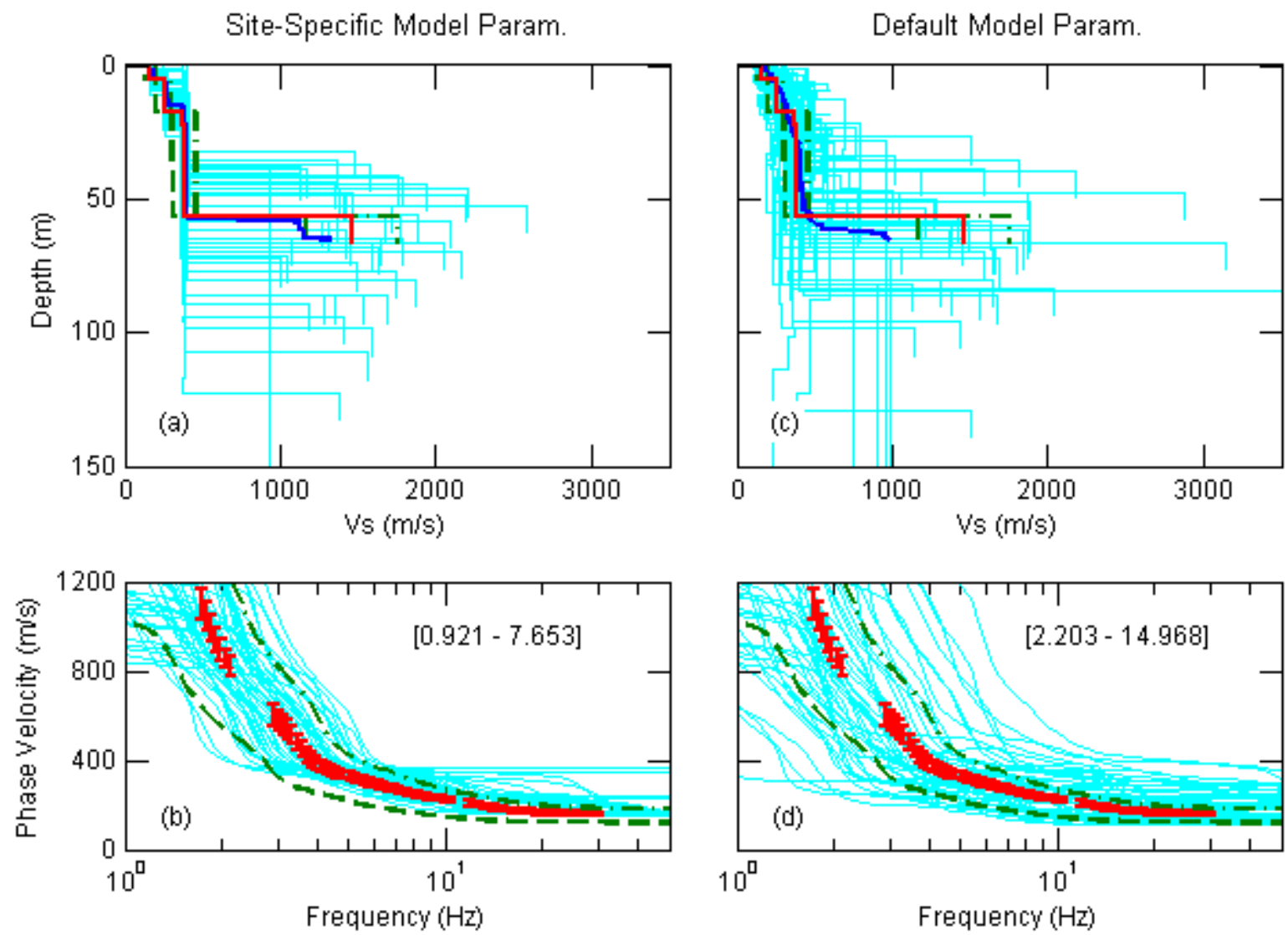

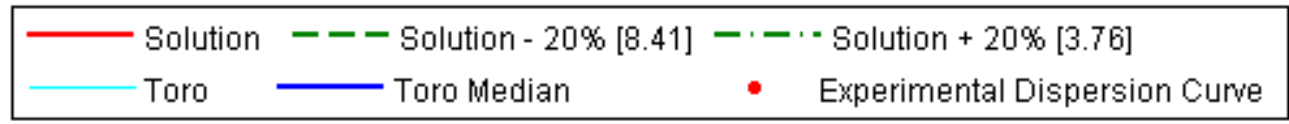

Figure 4: Fifty Vs profiles developed using the Toro (1995) randomization model and their corresponding theoretical dispersion curves with: $(a, b)$ site-specific Toro model parameters and (c, d) default/recommended Toro model parameters. Note that the numbers in brackets represent dispersion misfit values. Also shown are the theoretical dispersion curves and the Vs profiles corresponding to the solution profile $+/-20 \%$.

\section{$\underline{\text { Linear-Elastic Transfer Functions and the H/V Peak }}$}

Similar to the experimental dispersion curve, the experimental horizontal-to-vertical (H/V) spectral ratio curve contains valuable information regarding small-strain wave propagation and site resonance. Numerous studies have demonstrated that if an H/V curve exhibits a well-defined peak, then this peak approximately coincides with the fundamental shear wave resonant frequency of the site, although the magnitudes are poorly correlated (e.g., Lachez and Bard 1994 [34], Lermo and Chavez-Garcia 1994 [35]). Thus, by comparing the theoretical linear-elastic shear wave transfer function for a candidate Vs profile to the experimental H/V curve, it is possible to make judgements regarding which candidate Vs profiles are appropriate for use in seismic site response analyses. While it is possible to perform a joint inversion of surface wave dispersion data and the peak frequency of the H/V curve in Geopsy (Wood et al. 2014 [36], Bonnefoy-Claudet et al. 2006 [37]), we were not able to do this at blind-study Site 4 because the 
$\mathrm{H} / \mathrm{V}$ data was not initially provided to participants. However, it is valuable to consider how the experimental H/V curve coincidentally compares with the linear elastic transfer functions for the Vs profiles under consideration.

Figure 5a though 5f show the linear-elastic transfer functions for the Vs profiles obtained using each layering ratio in comparison to the experimental H/V curve for Site 4 . In order to calculate the transfer functions, small-strain damping values for each layer were assigned using the Darendeli (2001) [38] modulus reduction and damping curve relationships. It can be seen that the fundamental resonant frequency obtained from the transfer functions coincides with the H/V peak at $1.6 \mathrm{~Hz}$ for almost all Vs profiles determined from surface wave inversions, including those from the presumably poorly-parameterized layering ratios of 1.2 and 5.0. While there are a few outliers for a layering ratio of 1.5 , the majority of profiles have a resonant frequency between approximately 1.5 and $1.7 \mathrm{~Hz}$. Despite the fact that the resonant frequencies are similar for all inversion profiles, the transfer function amplitudes at this frequency vary considerably, with Fourier amplitude ratios ranging from less than 4 to greater than 10 . This variability in amplitude is primarily due to the impedance contrast between bedrock and the overlying soil layers. Profiles with higher impedance contrasts, such as those corresponding to layering ratios of 3.0 and 5.0 (refer to Figure 2), have higher transfer function amplitudes. This underscores the epistemic uncertainty regarding the Vs of bedrock, which can be difficult to determine using surface wave methods (Garofalo et al. 2016 [9]).

The transfer functions for Vs profiles derived from the Toro randomization model exhibit significantly more variability with regards to the resonant frequency (refer to Figure $5 \mathrm{~g}$ and $5 \mathrm{~h}$ ). While the resonant frequencies match the $\mathrm{H} / \mathrm{V}$ peak for a number of the randomized Vs profiles, many do not. Half of the site-specific Toro profiles have a resonant frequency lower than 1.3 or greater than 2.0. Resonant frequencies are generally lower for the default Toro profiles, with half falling below 1.1 or above 1.8. If the fundamental shear wave resonant frequency of the site is expected to exhibit significant variability, then the variability exhibited by the Toro profiles in Figure $5 \mathrm{~g}$ and $5 \mathrm{~h}$ may be deemed to reasonably represent aleatory variability. However, if $\mathrm{H} / \mathrm{V}$ measurements across the site indicate that the fundamental frequency is relatively constant, then it is worth considering whether the variability seen in Figure $5 \mathrm{~g}$ and $5 \mathrm{~h}$ is excessive. If this is deemed to be the case, then the Toro (1995) [26] randomization can be repeated with a rejection criteria that automatically eliminates Vs profiles whose fundamental frequency is deemed to be too high or too low. It is worth noting that one or more three-component seismometers can quickly be deployed in a grid pattern to obtain $\mathrm{H} / \mathrm{V}$ measurements over a large footprint. Thus, the $\mathrm{H} / \mathrm{V}$ technique provides an efficient means of inferring the variability of the fundamental shear wave resonant frequency (assuming that the $\mathrm{H} / \mathrm{V}$ curve exhibits a well-defined peak). The resonant frequencies for the $+/-20 \%$ Vs profiles (refer to Figure $5 \mathrm{i}$ ) are 1.34 and $2.00 \mathrm{~Hz}$, respectively, for the softer and stiffer Vs profiles. 

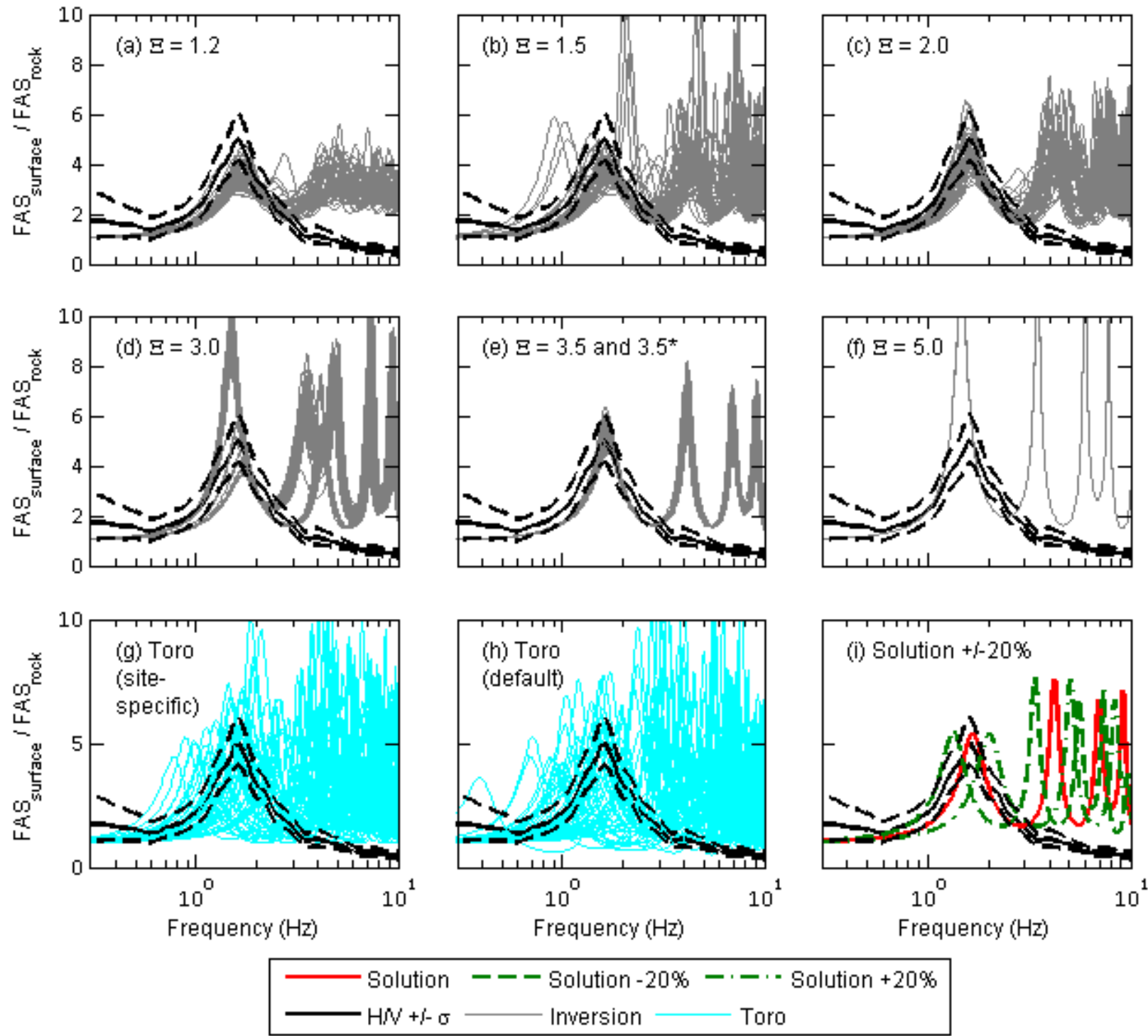

Figure 5: Linear-elastic transfer functions corresponding to Vs profiles obtained using surface wave inversion layering ratios of (a) 1.2, (b) 1.5, (c) 2.0, (d) 3.0, (e) 3.5 and 3.5*, and (f) 5.0. Also shown are transfer functions for Vs profiles obtained using (g) the site-specific Toro (1995) randomization model; (h) the default Toro (1995) randomization model; and (i) the solution $+/-$ $20 \%$.

\section{Equivalent-Linear Site Response Analyses}

Both low- and high-intensity equivalent-linear site response analyses were performed on the candidate Vs profiles, including: (1) the solution Vs profile, 350 Vs profiles derived from surface wave inversion, (2) the two upper/lower range Vs profiles, and (3) the $100 \mathrm{Vs}$ profiles statistically derived using the Toro (1995) [26] randomization procedure. The Boore and Atkinson (2008) [39] ground motion prediction equation (GMPE) was used to develop a target spectrum for the selection of reasonably consistent input rock ground motions. The target was computed using the following assumptions: a moment magnitude $\left(\mathrm{M}_{\mathrm{W}}\right)$ of 7.5 , a Joyner-Boore 
distance $\left(\mathrm{R}_{\mathrm{JB}}\right)$ of $15 \mathrm{~km}$, and an average Vs in the top $30 \mathrm{~m}\left(\mathrm{~V}_{\mathrm{S} 30}\right)$ of $1300 \mathrm{~m} / \mathrm{s}$, which is the upper limit for this GMPE. A $V_{\mathrm{S} 30}$ value of $1300 \mathrm{~m} / \mathrm{s}$ was chosen for the target spectrum because the majority of the bedrock velocities for the candidate Vs profiles ranged from approximately 1,000 to $2,000 \mathrm{~m} / \mathrm{s}$ (refer to Figures 2 and 4). While some profiles approached or exceeded 3,000 $\mathrm{m} / \mathrm{s}$, these bedrock velocities were less frequent. After developing a target spectrum, the PEER NGA-West 2 database (Ancheta et al. 2015 [40]) was used to develop a library of 40 ground motions with $\mathrm{M}_{\mathrm{W}}$ between 6.2 and 7.6, $\mathrm{R}_{\mathrm{JB}}$ between 1.8 and $65 \mathrm{~km}$, and $\mathrm{V}_{\mathrm{S} 30}$ between 770 and 2,000 m/s. The SigmaSpectra software (Kottke and Rathje 2008 [41], Kottke and Rathje 2012 [42]) was used to select and scale eight ground motions that, on average, matched the shape and amplitude of the target response spectrum. No conditions were imposed upon the variability of the selected ground motions (i.e., the motions were not scaled to match a target standard deviation).

In order to study the influence of earthquake intensity, the input ground motions were subsequently re-scaled to achieve average peak ground accelerations (PGA) of 0.05 and $0.30 \mathrm{~g}$. Analyses performed using these re-scaled GMs are referred to, respectively, as the "lowintensity" and "high-intensity" site response analyses for the remainder of the paper. Note that in practice, different target spectra and input ground motions would be used for the low- and highintensity site response analyses because spectral amplitudes do not necessarily scale linearly. However, the objective of this study is to assess differences in site response resulting from differences in the input Vs profile. Thus, the same GMs were used for consistency and simplicity. Also note that no attempts were made to variably-scale the input ground motions in order to account for significant differences in the bedrock impedance ratio for some of the Vs profiles (e.g., Figure $2 l$ ). Rather, all Vs profiles, regardless of bedrock Vs, were subject to the same suites of low- and high-intensity GMs. While not ideal, all Vs profiles had bedrock Vs consistent with fairly competent rock conditions and it would have been challenging to rescale GMs to account for specific bedrock velocity contrast in so many candidate profiles.

Equivalent-linear site-response analyses were performed using Matlab codes developed at the University of Texas (George Zalachoris, personal communication, 2014). These codes allowed the analyses to run in batch mode, looping through sets of candidate Vs profiles using eight GMs per profile. The Matlab code included auto-discretization of the layered earth model, which subdivided the major layers in the Vs profiles into sub-layers so that numerical filtering below $50 \mathrm{~Hz}$ would not be problematic. The code has been verified in the past (Griffiths et al. 2016b [19]) by comparing amplification factors and pseudo-acceleration response spectra with those computed using DEEPSOIL v5.1 (Hashash et al. 2012 [43]). The non-linear properties of each soil layer were set using the depth/confining pressure-dependent normalized modulus reduction $\left(\mathrm{G} / \mathrm{G}_{\mathrm{max}}\right)$ and damping (D) relationships proposed by Darendeli (2001) [38]. For ease and consistency, all layers were assumed to be non-plastic $(\mathrm{PI}=0)$ and normally-consolidated $(\mathrm{OCR}=1)$.

\section{Low-Intensity Input Ground Motions}

A median pseudo-acceleration response spectrum (simply referred to as a response spectrum hereafter) was computed for each candidate Vs profile using the eight individual response spectra resulting from the eight input ground motions. The median response spectra for all Vs profiles subjected to the low intensity input ground motions ( $0.05 \mathrm{~g}$ average PGA) are shown in Figure 6. For the inversion Vs profiles (Figure 6a through 6f), most response spectra are in good 
agreement with the response spectrum of the true solution profile. Vs profiles associated with a layering ratio of 3.5 and $3.5^{*}$ not only have the lowest dispersion misfit values $(0.026$ to 0.135 ; refer to Figure 2), but their response spectra also best match the response spectrum of the solution Vs profile, suggesting that relatively low dispersion misfit values are correlated with accurate site response predictions. However, it is also clear that the relationship between dispersion misfit and accuracy of site response is not perfect. While the Vs profiles associated with a layering ratio of 3.0 have relatively low dispersion misfit values $(0.10$ to 0.17$)$, the associated response spectra overestimate the spectral acceleration (SA) by as much as 85 percent at a period of $0.35 \mathrm{~s}$. This is due to the large impedance contrast between the bedrock and the overlying soil layers (see Figure $2 \mathrm{~h}$ ). On the other hand, the Vs profiles for a layering ratio of 1.2 were found to be under-constrained with relatively high dispersion misfit values ( 0.28 to 0.46$)$, yet their response spectra better match the solution. Similarly, Vs profiles for a layering ratio of 5.0 were found to be over-constrained with relatively high misfit values $(0.25)$, yet their SAs better match the solution than those for a layering ratio of 3.0. This indicates that while dispersion misfit may be useful for making relative judgements of the quality of Vs profiles resulting from inversion, it should not be used as the only basis for selecting candidate Vs profiles for site response. The shear wave velocity of the bedrock layer should also be carefully considered and constrained, if possible, by geology and/or other information (e.g., borehole Vs measurements).

The median response spectra for the $+20 \%$ Vs profile is generally in good agreement with the solution (refer to Figure 6), however, it does over-predict SA by about $40 \%$ at $0.21 \mathrm{~s}$. The $20 \%$ profile under-predicts the SA by about 20 to $40 \%$ at periods ranging from 0.01 to $0.17 \mathrm{~s}$, but is in good agreement at longer periods. The fact that the $+20 \%$ Vs profile is in better agreement with the solution than the $-20 \%$ profile underscores the influence of soil nonlinearity, even for relatively low-intensity input GMs.

Similar to the linear elastic transfer functions, the response spectra associated with the Toro profiles (Figure $6 \mathrm{~g}$ and $6 \mathrm{~h}$ ) exhibit significantly more variability than those associated with the inversion or $+/-20 \%$ profiles. The response spectra associated with the Toro profiles generally under-estimate SA. While this under-prediction is more pronounced for the default Toro profiles than for the site-specific Toro profiles, it is quite significant in both cases. As stated previously, the theoretical dispersion data for the Toro profiles poorly matches the experimental dispersion data (with misfits ranging from 0.92 to 15 ) and the fundamental resonant frequencies generally do not match the experimental $\mathrm{H} / \mathrm{V}$ peak. Thus, while the relationship between dispersion misfit and accuracy in site response prediction is not perfect, these results suggest that high dispersion misfit values are correlated with poor prediction of site response. Accordingly, if a site signature is robustly determined via surface wave surveys and $\mathrm{H} / \mathrm{V}$ measurements over the area of interest, and if the candidate Vs profiles fail to capture this site signature, it is worth questioning their use for quantifying uncertainty associated with the site response predictions. 

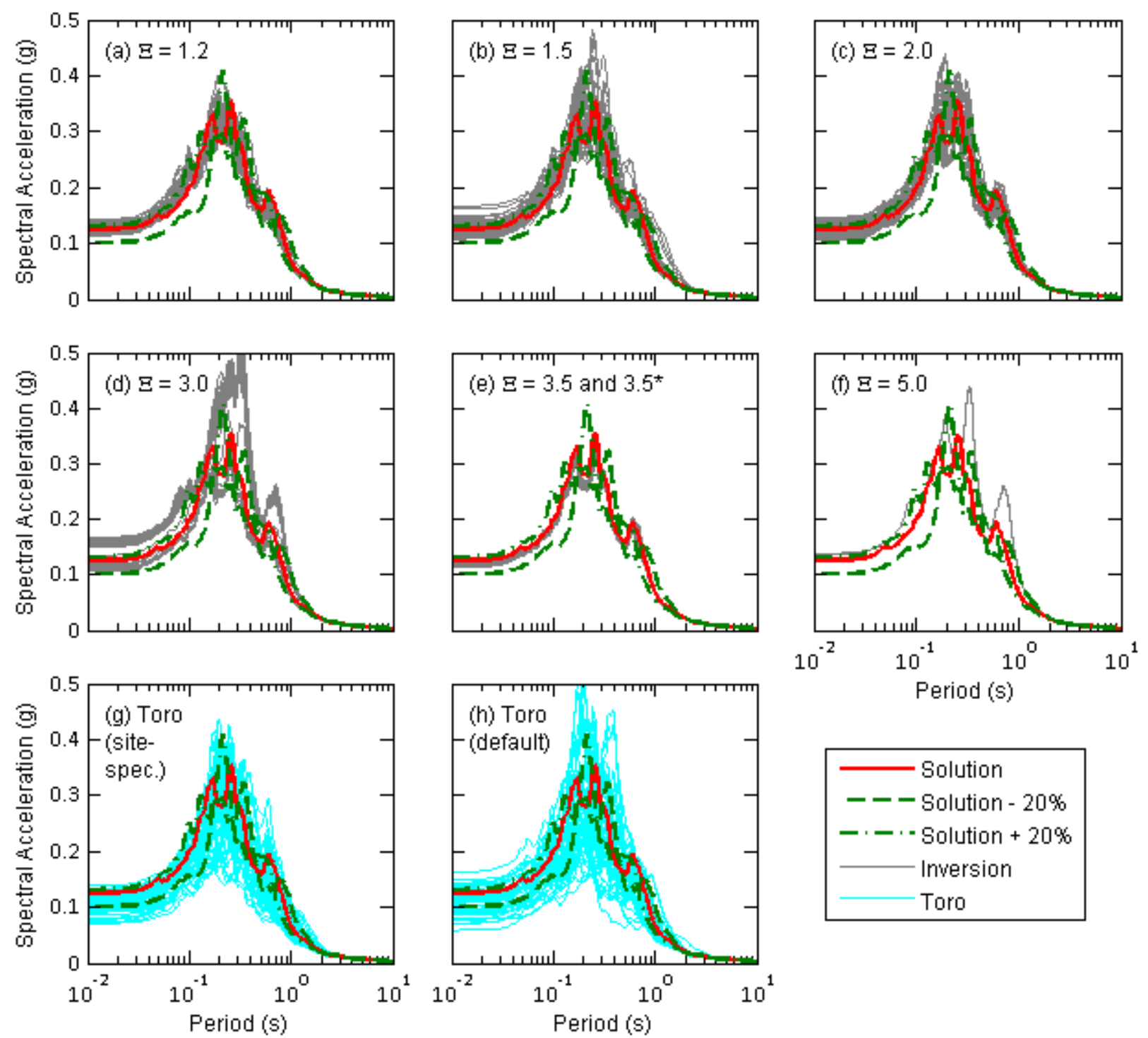

Figure 6: Median response spectra obtained from low-intensity equivalent-linear site response analyses using a suite of eight low-intensity input ground motions scaled to an average PGA of $0.05 \mathrm{~g}$ and Vs profiles from layering ratios of: (a) 1.2, (b) 1.5, (c) 2.0, (d) 3.0, (e) 3.5 and 3.5*, and (f) 5.0; and those from the Toro (1995) randomization model with: (g) the site-specific parameters, and (h) default parameters. The response spectra for the solution Vs profile and the solution Vs profile $+/-20 \%$ are shown in all sub-plots for comparison.

\section{High-Intensity Input Ground Motions}

The median response spectra for all Vs profiles based on the high-intensity input ground motions ( $0.30 \mathrm{~g}$ average PGA) are shown in Figure 7 . The response spectra for the inversion Vs profiles are shown in Figure 7a through $7 \mathrm{f}$. It can be seen that the response spectra for layering ratios of $2.0,3.0,3.5,3.5^{*}$, and 5.0 all match the response spectrum from the solution profile relatively well. Again, the response spectra for layering ratios of 3.5 and $3.5^{*}$, which have the lowest dispersion misfit values, best match the solution. Interestingly, a good match of the 
solution response spectrum based on the low-intensity ground motions does not necessarily result in an equally good agreement with the solution response spectrum for the high-intensity GMs. For example, the response spectra for layering ratios of 1.2 and 1.5 over-predict the solution SA for the high-intensity GMs, but reasonably predict the solution SA for the low-intensity GMs. Conversely, the response spectra for a layering ratio of 3.0 is in much better agreement with the solution for the high-intensity GMs. These differences are caused by the non-linear soil behavior induced by the high-intensity GMs. Specifically, the inversion Vs profiles with less significant velocity contrasts layer-to-layer (i.e., layering ratios 1.2 and 1.5) do not generate as high of shear strains at layer interfaces. Thus, more energy gets through to the ground surface and their SAs are, on average, significantly higher than for the solution Vs profile, which contains a significant impedance contrast. The exact opposite is true for all inversion Vs profiles with more significant velocity contrasts (i.e., layering ratios of 2.0, 3.0, 3.5 and 5.0). Meaning, high shear strains and significant soil nonlinearity occur at the layer boundaries where large impedance contrasts exist, limiting the amount of energy that arrives at the ground surface. Regardless, all of the inversion Vs profiles yield significantly less variability in SA than the upper/lower range profiles and the randomized profiles. When comparing the results from Figure 6 and Figure 7, it is evident that the under- and over-prediction associated with the $+/-20 \%$ Vs profiles is much more significant for the high-intensity GMs. The same is also true for the Toro Vs profiles, particularly those developed using the default parameters.

Figure 8 shows the median response spectra for each set of 50 inversion Vs profiles and each set of 50 Toro Vs profiles (i.e., the median of medians for each set) along with those for the $+/-20 \%$ Vs profiles and solution Vs profile subject to high-intensity GMs. It can be seen that response spectra for the $+/-20 \%$ Vs profiles significantly over/under-predict SA across most periods, with maximum errors up to $60 \%$ between periods of 0.2 and $0.3 \mathrm{~s}$. The Toro Vs profiles also result in poor site response estimates for high-intensity GMs, generally under predicting SA at most periods. The default Toro profiles exhibit greater error $(20 \%-50 \%$ at periods $<1.0 \mathrm{~s})$ than the site-specific profiles (maximum errors < 30\%). As noted earlier, the default Toro profiles were developed using the generic $\sigma_{\operatorname{lnVs}}$ values provided in Toro (1995) [26]. Although not shown here, it should be noted that when the site-specific $\sigma_{\operatorname{lnVs}}$ recommended in Stewart et al. (2014) [33] is used, the results are quite similar to those obtained using the randomization parameters that we developed for this particular site using the Vs profiles derived from surface wave inversions (referred to throughout the paper as the "site-specific Toro profiles"). Thus, we further the recommendation of Stewart et al. (2014) [33] that the site-specific $\sigma_{\ln V s}$ values be used in lieu of the generic values when lateral variability is not expected to be significant.

For both suites of Toro profiles the under predictions of SA are greatest at the softened natural period of the site, where site response estimates are critical. It is worth noting that, because the softened natural site period varies considerably for the site-specific and default Toro profiles, the site periods for individual response spectra are essentially smoothed-out and not well represented by the median response spectrum. This suggests that it may be best to consider individual response spectra from each suite rather than a statistical representation (e.g., the median or mean) of the suite. Nonetheless, it is clear from Figure $7 \mathrm{~g}$ and $7 \mathrm{~h}$ that the majority of individual response spectra underestimate the SA associated with the site period. Thus, the under-prediction shown in Figure 8 cannot be attributed solely to smoothing/averaging.

Remarkably, the visually-variable Vs profiles determined directly from inversion have SA values, on average, that are very similar to the solution Vs profile. Indeed, if the results from layering ratios of 1.2 and 5.0 are neglected due to poor parameterization (refer to discussion 
above), the remaining surface wave inversion results rarely deviate by more than $10 \%$ from the solution.
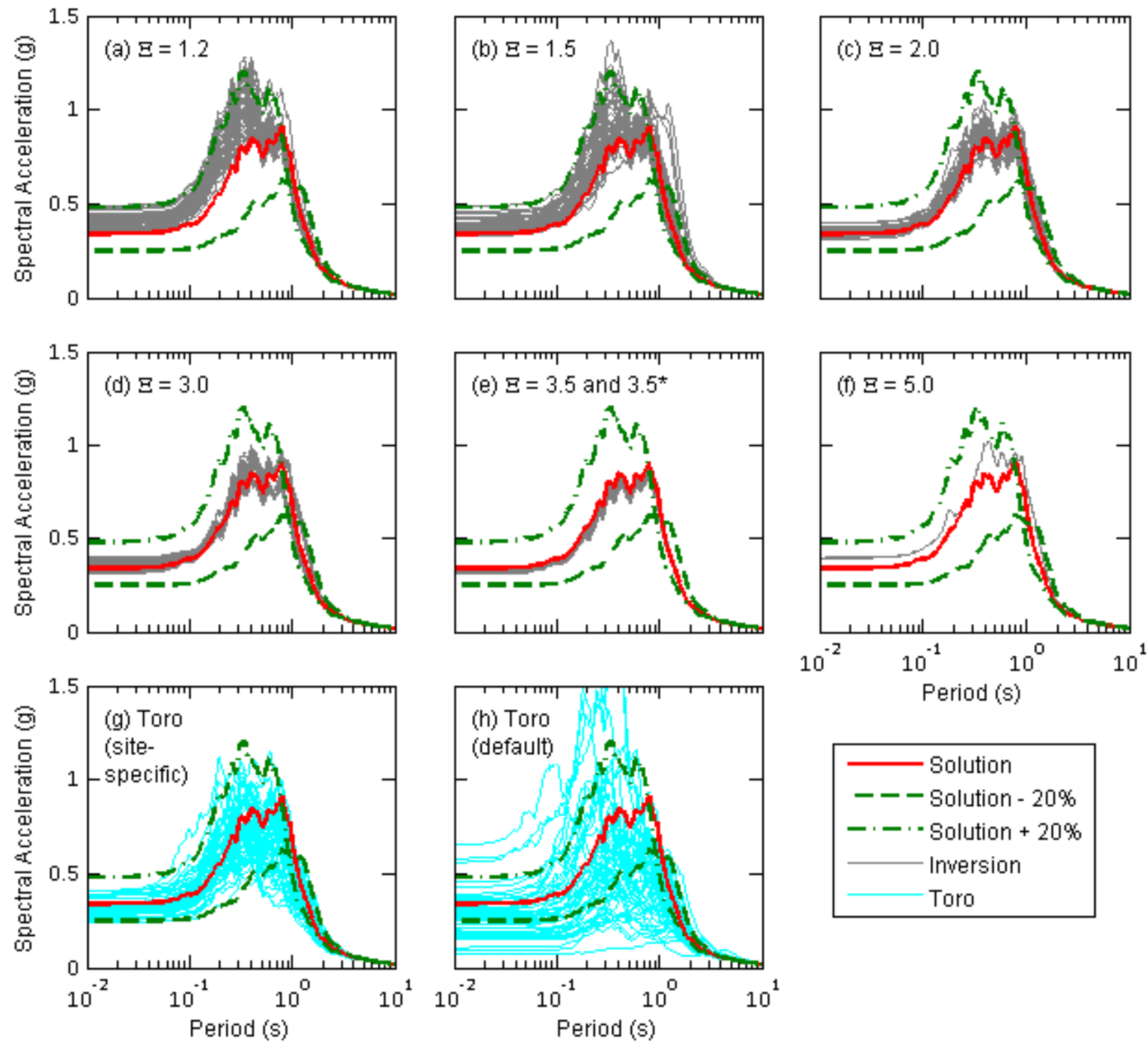

Period (s)

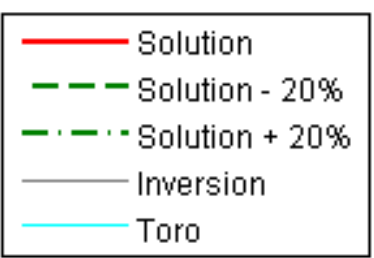

Figure 7: Median response spectra obtained from high-intensity equivalent-linear site response analyses using a suite of eight high-intensity input ground motions scaled to an average PGA of $0.3 \mathrm{~g}$ and Vs profiles from layering ratios of: (a) 1.2, (b) 1.5, (c) 2.0, (d) 3.0, (e) 3.5 and 3.5*, and (f) 5; and those from the Toro (1995) randomization model with: (g) the site-specific parameters, and (h) default parameters. The response spectra for the solution Vs profile and the solution Vs profile $+/-20 \%$ are shown in all sub-plots for comparison. 

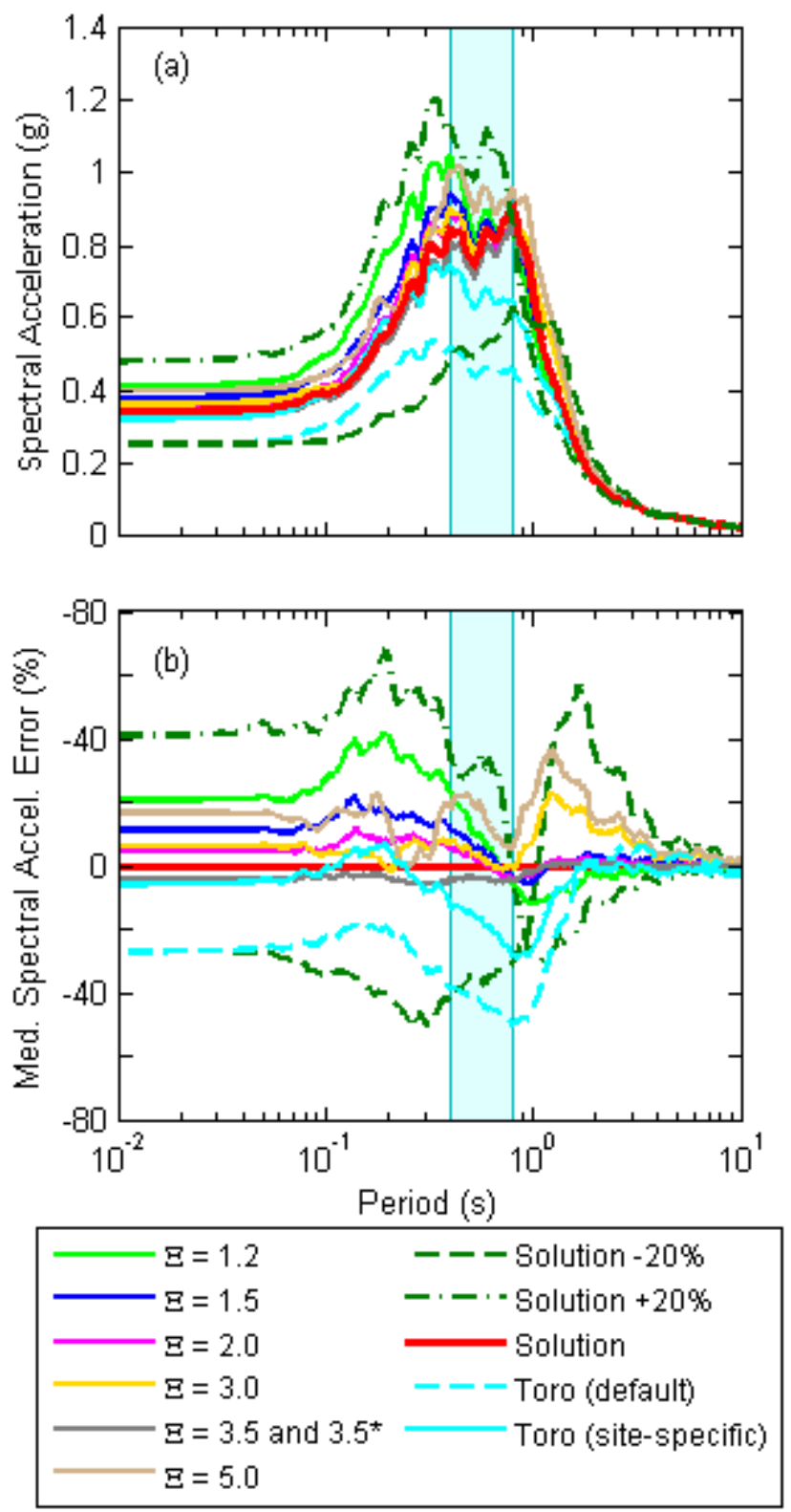

Figure 8: (a) Median response spectra for each set of 50 inversion Vs profiles and each set of 50 Toro Vs profiles (i.e., the median of medians for each set) along with those for the $+/-20 \% \mathrm{Vs}$ profiles and solution Vs profile subject to a suite of eight high-intensity input ground motions scaled to a PGA of $0.3 \mathrm{~g}$, and (b) Percent spectral acceleration error with respect to the response spectrum for the solution profile. Shaded region indicates the softened predominant period range of the solution Vs profile.

\section{$\underline{\text { Discussion }}$}

Although not perfect, there appears to be a relationship between how well the theoretical dispersion data for a Vs profile matches the experimental data and how well the associated response spectrum matches the response spectrum of the solution profile. In order to further 
investigate this topic, it is desirable to utilize a single parameter that quantifies how well or how poorly a response spectrum matches the solution response spectrum. A root-mean-square-error (RMSE) was computed for this purpose. The RMSE was computed as shown in Equation 2, where $\mathrm{SA}_{\mathrm{i}}$ is the spectral acceleration associated with a given profile at period $\mathrm{i}, \mathrm{SA}_{\text {solution, }}$ is the spectral acceleration associated with the solution profile at period $\mathrm{i}$, and $\mathrm{Np}$ is the number of discrete periods in the response spectra. In this study, all response spectra had 512 periods equally spaced on a logarithmic scale between 0.01 and $10 \mathrm{~s}$. Note that narrower period ranges (e.g. 0.1-10 s and 0.1-1 s) were also considered and found to produce similar results.

$$
R M S E=\sqrt{\frac{1}{N_{p}} \sum_{i=1}^{N_{p}}\left[\ln \left(S A_{i}\right)-\ln \left(S A_{\text {solution }, i}\right)\right]^{2}}
$$

The RMSE is plotted against the dispersion misfit for the low-intensity and high-intensity input GMs in Figure 9. While the trends are not perfect and scatter in the data is significant, it is clear that increases in dispersion misfit are generally accompanied by increases in RMSE for both the low- and high-intensity GMs. However, there are notable exceptions to this trend. As discussed earlier, the Vs profiles associated with a layering ratio of 3.0 result in relatively low misfit values and relatively high RMSE values for the low-intensity GMs. Conversely, there are a few site-specific Toro profiles whose RMSE values are comparable to the inversion profiles despite the fact that the misfit values are one to two orders of magnitude higher than the inversion profiles. Nonetheless, the dispersion misfit is generally a good indicator of the accuracy of the predicted site response at this particular site.

As discussed previously, the differences between Vs profiles derived from a single inversion parameterization are often much smaller than the differences between Vs profiles derived from different parameterizations (refer to Figure 2). Hence, when limited information is known at the site (particularly at significant depths), the non-uniqueness in Vs associated with various inversion parameterizations is a concern. However, the significantly different Vs profiles utilized in this study have been shown to yield similar site response estimates in virtually all cases. In order to further investigate this matter, 250 response spectra associated with layering ratios of 1.5 through $3.5^{*}$ were used to compute a single, lognormal median response spectrum with associated standard deviation $\left(\sigma_{\operatorname{lnSA}}\right)$. Results from layering ratios of 1.2 and 5.0 were excluded from this calculation due to their assumed poor-parameterizations, as discussed above. Similar to what was done for the computation of site-specific Toro parameters, each response spectrum was weighted by the inverse of the dispersion misfit, giving those profiles with a lower misfit more weight in the calculations. The same weighting system was utilized to compute a lognormal median response spectrum and associated standard deviation for the $50 \mathrm{Vs}$ profiles associated with the site-specific Toro parameters and the $50 \mathrm{Vs}$ profiles associated with the default Toro parameters.

The dispersion misfit-weighted lognormal median response spectra with $+/$ - one standard deviation values are provided in Figure 10. The weighted response spectra obtained from the inversion Vs profiles are shown in Figure 10a and 10b for the low- and high-intensity input GMs, respectively. In both cases, the lognormal median response spectrum is almost identical to the response spectrum of the solution Vs profile. This finding is quite remarkable, given the apparent non-uniqueness in the inversion Vs profiles. Moreover, the associated standard deviations are also quite low, particularly for the high-intensity GMs. Thus, while the input 
profiles may vary considerably, their associated misfit-weighted response spectra exhibit minimal variability and match that of the solution remarkably well.

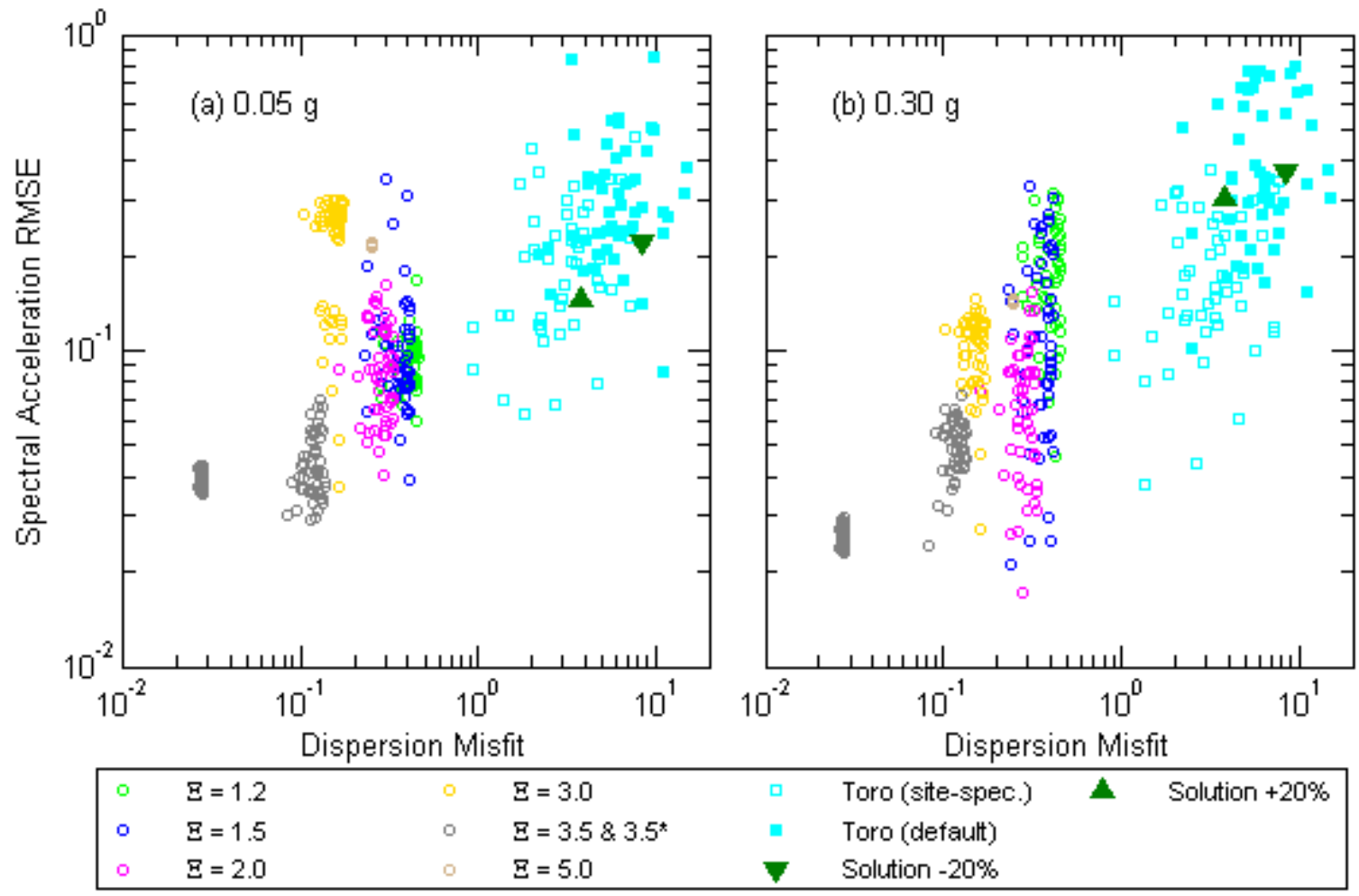

Figure 9: Spectral acceleration RMSE versus dispersion misfit for all Vs profiles considered. Response spectra were calculated using input ground motions scaled to an average PGA of (a) $0.05 \mathrm{~g}$ (i.e., low-intensity) and (b) $0.30 \mathrm{~g}$ (i.e., high-intensity). Note that the response spectrum corresponding to the solution Vs profile was used as a reference in the RMSE calculations.

The results corresponding to the site-specific Toro profiles are shown for the low- and high-intensity GMs in Figure 10c and 10d, respectively. For the low-intensity GMs (Figure 10c), the solution response spectrum generally better matches the plus-one standard deviation curve than the lognormal median. This indicates consistent under-prediction of the true site-response. For the high-intensity GMs (Figure 10d), the lognormal median response spectrum for the sitespecific Toro profiles matches the solution until approximately $0.30 \mathrm{~s}$ (i.e., the softened site period), above which the plus-one standard deviation curve better matches the solution.

As shown in Figure 10e and 10f, the lognormal median response spectrum for the default Toro profiles poorly matches the solution and significantly under-predicts SA at all periods for both the low- and high-intensity GMs. Moreover, the high-intensity plus-one standard deviation curve falls below the solution response spectrum at periods longer than 0.4 s (i.e., near the softened site period). This happens in spite of the fact that the standard deviation is quite large. Thus, the default Toro profiles yield significant variability in site response and severely underestimate the SA in the vicinity of the predominant period. These results show similar trends to those shown in Rathje et al. (2010) [2], who found that the median surface response spectrum decreases as more variability (i.e., increased $\sigma_{\operatorname{lnV} s}$ ) is incorporated into the randomization model. Thus, while it may appear conservative to assume higher variability in the Vs randomization, the resulting site response may in fact underestimate the "true" spectral accelerations. Again, the 
substantial variability and resulting smoothing-out of individual site periods contributes in part to the under-prediction associated with the median response spectrum for the site-specific and default Toro profiles.

Figure 11 shows the dispersion misfit-weighted standard deviation of the natural logarithm of SA $\left(\sigma_{\operatorname{lnSA}}\right)$ as a function of period for both the low- and high-intensity input GMs. Note that these standard deviations correspond to the response spectra shown in Figure 10. The $\sigma_{\operatorname{lnSA}}$ curves associated with the low-intensity input GMs are shown in Figure 11a. These curves are similar for the inversion profiles and for the site-specific Toro profiles, although the $\sigma_{\operatorname{lnSA}}$ curve associated with the inversion profiles shows local minima at periods of 0.13 and $0.53 \mathrm{~s}$. The $\sigma_{\operatorname{lnSA}}$ associated with the default Toro profiles is about 0.05 to 0.1 greater than that associated with the site-specific Toro profiles. In all cases $\sigma_{\operatorname{lnSA}}$ shows similar trends in terms of shape. Specifically, $\sigma_{\operatorname{lnSA}}$ is relatively constant at periods below $0.1 \mathrm{~s}$, reaches a maximum at or near the softened site period (shaded region), and decreases at periods longer than $1 \mathrm{~s}$. The amplitude and shape of the $\sigma_{\operatorname{lnSA}}$ curve for the default Toro Vs profiles are in good agreement with $\sigma_{\operatorname{lnSA}}$ curves developed from weak ground motions at the La Cienga site described in Li and Assimaki (2010) [3]. Similar to the default Toro Vs profiles described in this paper, they also used generic parameters to randomize about their base case Vs profile.

The $\sigma_{\operatorname{lnSA}}$ curves associated with the high-intensity input GMs are shown in Figure $11 \mathrm{~b}$. For the inversion and site-specific Toro profiles, the $\sigma_{\operatorname{lnSA}}$ curves associated with the highintensity GMs are slightly lower than for the low-intensity GMs (Figure 11a) and begin to drop off at longer periods (roughly $1.5-2 \mathrm{~s}$ versus $0.8-1 \mathrm{~s}$, respectively). This shift may be due in part to the fact that the softened site period is longer for the high intensity GMs. On the other hand, the $\sigma_{\operatorname{lnSA}}$ associated with the default Toro Vs profiles is substantially higher for the high-intensity GMs than for the low-intensity GMs. This demonstrates that the influence of Vs profile uncertainty on the overall site response uncertainty is site and ground motion dependent and may not always follow the same trends.

It is important to note that the reference/baseline profile used to develop all Toro profiles was equal to the solution profile and represents a best case scenario. Thus, if a different, less accurate reference profile had been used then the resulting site response predictions would deviate even more from the "true" site response. Of course, this assumes that a "true" site response exists, whereas at real sites the ground response may vary over the area of interest. The use of site-specific Vs randomization parameters developed directly from a large number of surface wave Vs profiles led to significantly more accurate and less variable site response estimates. Thus, the authors strongly recommend that site-specific Vs randomization parameters be developed whenever possible. High quality surface wave testing with rigorous inversions considering multiple parameterizations can be used for this purpose. When this is not possible, the authors recommend that the site-specific variability recommended in Stewart et al. (2014) [33] be used, unless extreme aleatory variability is expected at the site. The site response results obtained from the $+/-20 \mathrm{Vs}$ profiles are even more variable than those associated with those from Vs randomization for the high-intensity input ground motions. The fact that the upper/lower range and randomized Vs profiles yield significantly different site response than the solution profile and the Vs profiles determined directly from inversion should not be surprising given how poorly these profiles matched the experimental dispersion and $\mathrm{H} / \mathrm{V}$ data.

As noted earlier, if the peak of the experimental H/V curve and dispersion data can be demonstrated to represent a consistent signature of the site, then it is worth considering if Vs profiles that fail to capture this site signature are appropriate for use in site response. The results 
of this paper are not meant to suggest that the Toro (1995) [26] model is not useful for accounting for Vs uncertainty. Rather, the results suggest that analysts should be mindful about the appropriate use of this randomization model. At real sites, experimental dispersion data and $\mathrm{H} / \mathrm{V}$ curves measured over a large area may vary considerably or they may be extremely consistent. For the former situation, Vs profiles developed using the Toro (1995) [26] model may perform more favorably because the site signature itself exhibits significant variability. In either case, if sufficient measurements are conducted and a site signature can be established, then an analyst may choose to implement certain rejection criteria in the randomization process, whereby Vs profiles whose theoretical dispersion curves or fundamental frequencies deviate substantially from the site signature are rejected.
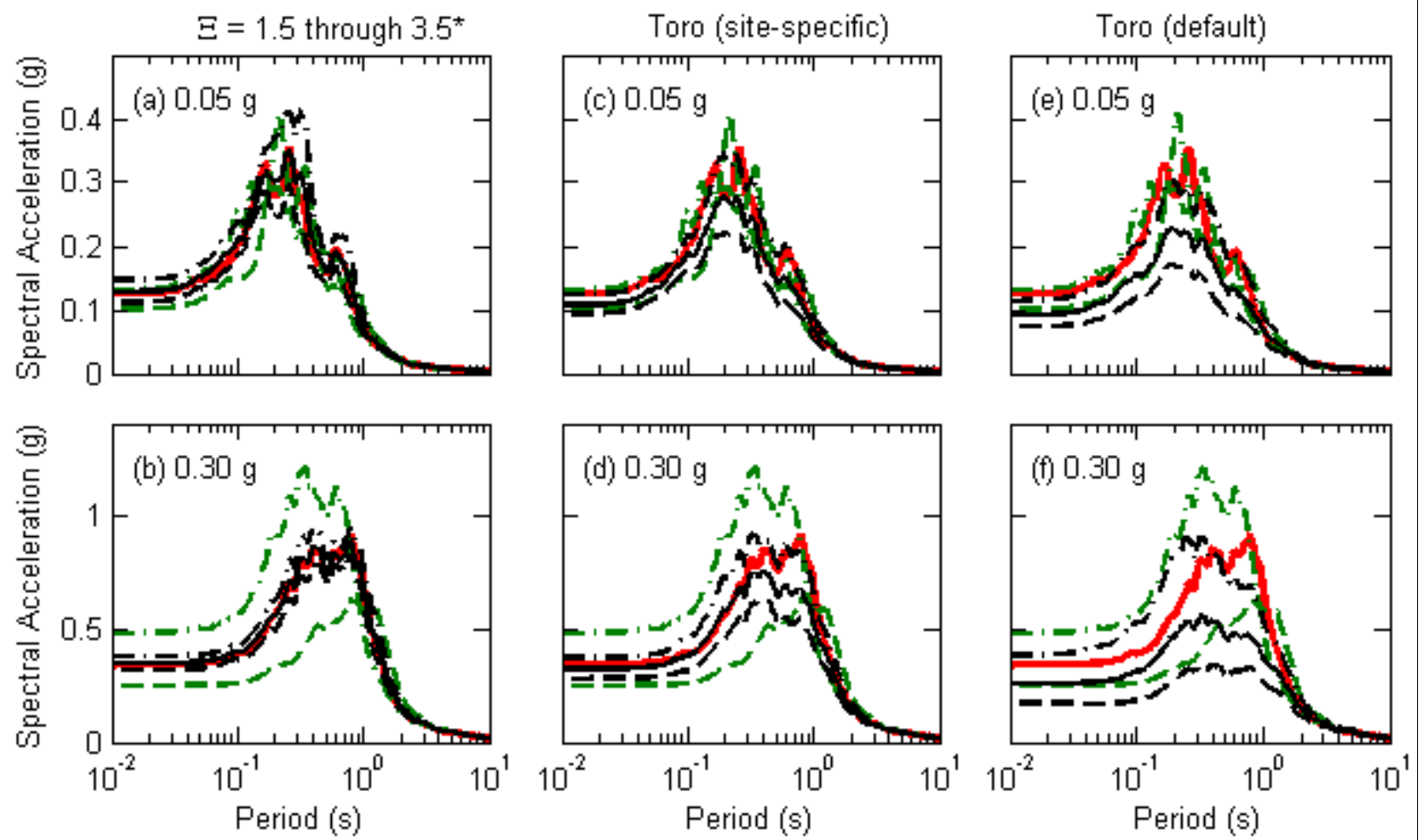

$$
\begin{aligned}
& \hline \text { Solution }--- \text { Solution }-20 \%-\cdot-\cdots \text { Solution }+20 \% \\
& \hline \text { Median }--- \text { Median }-\sigma_{\operatorname{InSA}}-\cdot-\cdot-\text { Median }+\sigma_{\operatorname{InSA}} \\
& \hline
\end{aligned}
$$

Figure 10: Dispersion misfit-weighted lognormal median response spectrum (solid line) with +/one standard deviation (dashed lines) for: $(\mathrm{a}, \mathrm{b})$ the combination of 250 inversion Vs profiles from layering ratios of 1.5, 2.0, 3.0, 3.5, and 3.5*; (c,d) 50 site-specific Toro profiles; and (e,f) 50 default Toro profiles. Response spectra were obtained using both low-intensity (a,c,e) and high-intensity (b,d,f) input ground motions. The response spectra for the solution Vs profile and the $+/-20 \%$ Vs profiles are shown for reference in all sub-plots. 

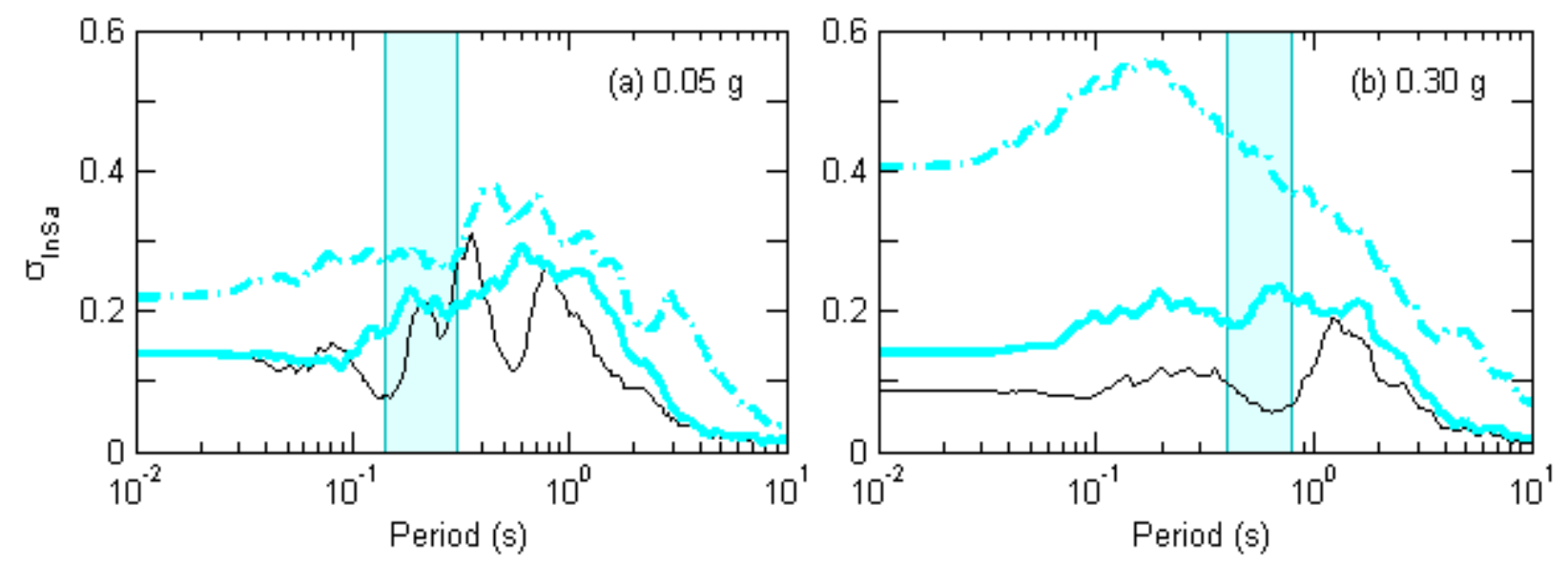

$\Xi=1.5$ through $3.5^{*} \longrightarrow$ Toro (site-specific) $-1=\cdots$ Toro (default)

Figure 11: Dispersion-misfit weighted standard deviation of the natural logarithm of surface spectral acceleration associated with (a) the low-intensity input ground motions and (b) the highintensity input ground motions. Note that the standard deviations correspond to the response spectra shown in Figure 10. Shaded regions indicate the softened predominant period range of the solution Vs profile.

\section{Conclusions}

Variability and accuracy of site response predictions performed using shear wave velocity profiles (Vs) derived from non-unique surface wave inversions and other commonly used statistical methods of accounting for epistemic uncertainty and aleatory variability in Vs have been considered. Despite visually-significant differences in the Vs profiles derived from surface wave inversions using different layering parameterizations, so long as their theoretical dispersion data fit the experimental dispersion data well, their dispersion misfit-weighted site response results were quite accurate with minimal variability. If a site signature can be established, then non-unique Vs profiles derived from surface wave inversion provide a means for accounting for Vs uncertainty in a rational manner, so long as they are obtained properly by systematically exploring various layering parameterizations.

Upper/lower range Vs profiles (e.g., mean +/- 20\%) commonly utilized to account for epistemic uncertainty did not fit the experimental dispersion data well and were found to significantly over/under-predict spectral accelerations (SA) for high-intensity input GMs. Many statistically-based, randomly-generated Vs profiles commonly utilized to account for aleatory variability also failed to fit the experimental dispersion data or $\mathrm{H} / \mathrm{V}$ curve and were found to yield inaccurate and highly-variable SA predictions, although the inclusion of site-specific Vs randomization model parameters derived from the surface wave inversion Vs profiles improved the results. While not perfect, a clear trend between dispersion misfit and error/variability in site response has been demonstrated. When attempting to realistically account for Vs uncertainty in site response, the use of Vs profiles that do not well-fit the experimentally-measured site signature (i.e., experimental dispersion data and $\mathrm{H} / \mathrm{V}$ curves measured over the area of the site) should be questioned. It should be noted that at many real sites the site signature may exhibit considerable variability and the randomized and upper/lower range Vs profiles may better capture the site signature and yield more reasonable site response estimates than they did for this semi-synthetic example with lesser uncertainty/variability. The results of this paper do not 
suggest that analysts should not consider upper/lower range or randomized Vs profiles in site response, rather, they suggest using a more thoughtful approach when developing these profiles. If the site signature has been robustly determined, it may be possible to develop rejection criteria, whereby Vs profiles that poorly capture the site signature are not considered in subsequent site response analyses.

\section{Acknowledgments}

This work was supported primarily by U.S. National Science Foundation (NSF) grant CMMI1261775. However, any opinions, findings, and conclusions or recommendations expressed in this material are those of the authors and do not necessarily reflect the views of NSF. The authors would also like to acknowledge and thank the organizing committee of the InterPacific project, which was formed under the Research \& Development Program SIGMA, funded by EDF, AEREVA, CEA, ENEL, and the CASHIMA project, funded by CEA, ILL and IITER Organization.

\section{$\underline{\text { References }}$}

[1] P. Bazzuro and C. Cornell, "Ground-Motion Amplification in Nonlinear Soil Sites with Uncertain Properties," Bulletin of the Seismological Society of America, vol. 94, no. 6, pp. 2090-2109, 2004.

[2] E. M. Rathje, A. R. Kottke and W. L. Trent, "Influence of Input Motion and Site Property Variabilities on Seismic Site Response Analysis," Journal of Geotechnical and Geoenvironmental Engineering, vol. 136, no. 4, pp. 607-619, 2010.

[3] W. Li and D. Assimaki, "Site- and Motion-Dependent Parametric Uncertainty of SiteResponse Analyses in Earthquake Simulations," Bulletin of the Seismological Society of America, vol. 100, no. 3, p. 954-968, 2010.

[4] S. Barani, R. D. Ferrari and G. Ferretti, "Influence of Soil Modeling Uncertainties on Site Response," Earthquake Spectra, vol. 29, no. 3, pp. 705-732, 2013.

[5] American Society of Civil Engineers (ASCE), "Minimum design loads for buildings and other structures," ASCE Standard ASCE/SEI 7-10, ASCE, Reston, Virginia.

[6] American Association of State Highway and Transportation Officials (AASHTO), Guide Specifications for LRFD Seismic Bridge Design, 2nd ed., Washington, DC: AASHTO, 2011.

[7] Electric Power Research Institute (EPRI), "Seismic Evaluation Guidance: Screening, Prioritization and Implementation Details (SPID) for the Resolution of Fukushima NearTerm Task Force Recommendation 2.1: Seismic," EPRI, Report 1025287, Palo Alto, CA, 2012.

[8] S. C. Griffiths, B. R. Cox, E. M. Rathje and D. P. Teague, "A Surface Wave Dispersion Approach for Evaluating Statistical Models that Account for Shear Wave Velocity Uncertainty," Journal of Geotechnical and Geoenvironmental Engineering, vol. 82, pp. 222-240, DOI: 10.1061/(ASCE)GT.1943-5606.0001552, 2016a. 
[9] F. Garofalo, S. Foti, F. Hollender, P. Y. Bard, C. Cornou, B. R. Cox, M. Ohrnberger, D. Sicilia, M. Asten, G. DiGiulio, T. Forbriger, B. Guiller, K. Hayashi, A. Martin, S.

Matsushima, D. Mercerat, V. Poggi and H. Yamanaka, "InterPACIFIC Project: Comparison of Invasive and Non-Invasive Methods for Seismic Site Characterization. Part I: IntraComparison of Surface Wave Methods," Soil Dynamics and Earthquake Engineering, vol. 82, pp. 222-240, doi:10.1016/j.soildyn.2015.12.010, 2016a.

[10] F. Garofalo, S. Foti, F. Hollender, P. Y. Bard, C. Cornou, B. R. Cox, A. Dechamp, M. Ohrnberger, V. Perron, D. Sicilia, D. Teague and C. Vergniault, "InterPACIFIC Project: Comparison of Invasive and Non-Invasive Methods for Seismic Site Characterization. Part II: Inter-Comparison Between Surface Wave and Borehole Methods," Soil Dynamics and Earthquake Engineering, vol. 82, pp. 241-254, doi:10.1016/j.soildyn.2015.12.009, $2016 \mathrm{~b}$.

[11] C. G. Lai, S. Foti and G. Rix, "Propagation of Data Uncertainty in Surface Wave Inversion," Journal of Environmental and Engineering Geophysics, vol. 10, no. 2, pp. 219-228, 2005.

[12] C. Cornou, M. Ohrnberger, D. M. Boore, K. Kudo and B. P. Y, "Derivation of structural models from ambient vibration array recordings: results from an international blind test," in 3rd International symposium on the effects of surface geology on seismic motion, vol. 2, Grenoble, pp. 1127-1219, 2009.

[13] B. R. Cox, C. M. Wood and D. P. Teague, "Synthesis of the UTexas1 Surface Wave Dataset Blind-Analysis Study: Inter-Analyst Dispersion and Shear Wave Velocity Uncertainty," in Geo-Congress 2014 Technical Papers, Atlanta, GA, pp. 850-859, 2014.

[14] G. DiGiulio, A. Savvaidis, M. Ohrnberger, M. Wathelet, C. Cornou, B. Knapmeyer-Endrun, F. Renalier, N. Theodoulidis and P. Y. Bard, "Exploring the model space and ranking a best class of models in surface-wave dispersion inversion: Application at European strongmotion sites," Geophysics, vol. 77, no. 3, pp. B147-B166, 2012.

[15] B. R. Cox and D. P. Teague, "Layering Ratios: A Systematic Approach to the Inversion of Surface Wave Data in the Absence of A-priori Information," Geophysical Journal International, (submitted February 2016).

[16] S. Foti, C. Comina, D. Boiero and L. Socco, "Non-uniqueness in surface-wave inversion and consequences on seismic site response analyses," Soil Dynamics and Earthquake Engineering, vol. 29, pp. 982-993, 2009.

[17] J. Boaga, G. Vignoli and G. Cassiani, "Shear wave profiles from surface wave inversion: the impact of uncertainty on seismic site response analysis," Journal of Geophysics and Engineering, vol. 8, pp. 162-174, 2011.

[18] R. S. Jakka, N. Roy and H. R. Wason, "Implications of Surface Save Data Measurement Uncertainty on Seismic Ground Response Analysis," Soil Dynamics and Earthquake Engineering, Vols. 61-62, pp. 239-245, 2014a.

[19] S. C. Griffiths, B. R. Cox, E. M. Rathje and D. P. Teague, "Mapping Dispersion Misfit and Uncertainty in Vs Profiles to Variability in Site Response Estimates," Journal of Geotechnical and Geoenvironmental Engineering, DOI: 10.1061/(ASCE)GT.19435606.0001553, 2016b.

[20] C. Comina and S. Foti, "Discussion on 'Implications of Surface Save Data Measurement 
Uncertainty on Seismic Ground Response Analysis' by Jakka et al.," Soil Dynamics and Earthquake Engineering, 2014.

[21] R. S. Jakka, N. Roy and H. R. Wason, "Reply on 'Implications of Surface Save Data Measurement Uncertainty on Seismic Ground Response Analysis'," Soil Dynamics and Earthquake Engineering, 2014b.

[22] J. Boaga, G. Vignoli and G. Cassiani, "Reply to comment on 'Shear wave profiles from surface inversion: the impact of uncertainty on seismic site response analysis'," Journal of Geophysics and Engineering, vol. 9, pp. 244-246, 2012.

[23] L. V. Socco, S. Foti and C. Comina, "Comment on 'Shear wave profiles from surface inversion: the impact of uncertainty on seismic site response analysis'," Journal of Geophysics and Engineering, vol. 9, pp. 241-243, 2012.

[24] M. Wathelet, D. Jongmans and M. Ohrnberger, "Surface-wave inversion using a direct search algorithm and its application to ambient vibration measurements," Near Surface Geophysics, vol. 2, no. 4, pp. 211-221, 2004.

[25] N. Matasovic and Y. Hashash, "NCHRP Synthesis 428: Practices and Procedures for SiteSpecific Evaluations of Earthquake Ground Motions, A Synthesis of Highway Practice," National Cooperative Highway Research Program of the Transportation Research Board, Washington, D.C., 2012.

[26] G. Toro, "Probabilistic models of the site velocity profiles for generic and site-specific ground-motion amplification studies," Technical Report No. 779574, Brookhaven National Laboratory, Upton, N.Y., pp. 147, 1995.

[27] E. Thompson, L. Baise, Y. Tanaka and R. Kayen, "A taxonomy of site response complexity," Soil Dynamics and Earthquake Engineering, vol. 41, pp. 32-43, 2012.

[28] W. T. Thomson, "Transmission of elastic waves through a stratified solid medium," Journal of Applied Physics, vol. 21, pp. 89-93, 1950.

[29] N. A. Haskell, "The dispersion of surface waves on multilayered media," Bulletin of Seismological Society of America, vol. 43, pp. 17-34, 1953.

[30] J. W. Dunkin, "Computation of modal solutions in layered, elastic media at high frequencies," Bulletin of the Seismological Society of America, vol. 55, pp. 335-358, 1965.

[31] L. Knopoff, "A matrix method for elastic wave problems," Bulletin of the Seismological Society of America, vol. 54, pp. 431-438, 1964.

[32] A. Kottke and E. Rathje, "Technical Manual for Strata," Report No. 2008/10, Pacific Earthquake Engineering Research Center, Berkeley, California.

[33] J. Stewart, K. Afshari and Y. Hashash, "Guidelines for Performing Hazard-Consistent OneDimensional Ground Response Analysis for Ground Motion Prediction," Pacific Earthquake Engineering Research Center, Berkely, California, 2014.

[34] C. Lachet and P. Y. Bard, "Numerical and theoretical investigations on the possibilities and limitations of Nakamura's technique," Journal of Physics of the Earth, vol. 42, pp. 337-397, 1994.

[35] J. Lermo and F. J. Chavez-Garcia, "Site effects evaluation using spectral ratios with only 
one station," Bulletin of the Seismological Society of America, vol. 83, pp. 1574-1594, 1993.

[36] C. Wood, T. Ellis, D. Teague and B. Cox, "Analyst I: Comprehensive Analysis of the UTexas1 Surface Wave Dataset," in Geo-Congress 2014 Technical Papers, Atlanta, GA, pp. 820-829, 2014.

[37] S. Bonnefoy-Claudet, C. Cornou, P. Y. Bard, F. Cotton, P. Moczo, J. Kristek and D. Fah, "H/V ratio: a tool for site effects evaluation. Results from 1-D noise simulations," Geophysical Journal International, vol. 167, pp. 827-837, 2006.

[38] M. B. Darendeli, "Development of A New Family of Normalized Modulus Reduction and Material Damping Curves," PhD Dissertation, The University of Texas, Austin, TX, 2001.

[39] D. A. G. Boore, "Ground-motion Prediction Equations for the Average Horizontal Component of PGA, PGV, and the 5\%-damped PSA at spectral periods between $0.01 \mathrm{~s}$ and 10.0 s," Earthquake Spectra, vol. 24, pp. 99-138, 2008.

[40] T. Ancheta, R. Darragh, J. Stewart, E. Seyhan, W. Silva, B. Chiou, K. Wooddell, R. Graves, A. Kottke, D. Boore, T. Kishida and J. Donahue, "NGA-West2 database," Earthquake Spectra, vol. 30, pp. 989-1005, 2014.

[41] A. Kottke and E. Rathje, "A Semi-Automated Procedure for Selection and Scaling of Recorded Earthquake Motions for Dynamic Analysis," Earthquake Spectra, vol. 24, no. 4, pp. 911-932, 2008.

[42] A. R. Kottke and E. M. and Rathje, "Technical Manual for SigmaSpectra," 2012.

[43] Y. M. Hashash, D. R. Groholski, C. A. Phillips, D. Park and M. Musgrove, "DEEPSOIL 5.1, User Manual and Tutorial," 2012. 


\begin{tabular}{|c|c|c|c|c|c|c|c|c|}
\hline \multicolumn{3}{|c|}{ Vs statistical parameters } & \multicolumn{3}{|c|}{ Layering parameters } & \multicolumn{3}{|c|}{ Depth to bedrock parameters } \\
\hline & Default ${ }^{A}$ & Site-specific & & Default & Site-specific & & Default $^{B}$ & Site-specific \\
\hline$\sigma_{\ln V s}$ & 0.31 & 0.04 to 0.22 & a & 1.98 & 2.4 & base-case $[\mathrm{m}]$ & 56 & 56 \\
\hline$\Delta$ & 3.9 & default & $\mathrm{b}$ & 10.86 & 4.0 & $\sigma_{\ln Z r o c k}$ & 0.33 & 0.33 \\
\hline $\mathrm{d}_{0}$ & 0 & default & c & -0.89 & -1.15 & & & \\
\hline $\mathrm{b}$ & 0.344 & default & & & & & & \\
\hline$\rho_{0}$ & 0.99 & default & & & & & & \\
\hline$p_{200}$ & 0.98 & default & & & & & & \\
\hline
\end{tabular}

A. Default for sites with a $V_{s 30}$ ranging from 180 to $360 \mathrm{~m} / \mathrm{s}$

B. No default/recommended valves provided in Toro (1995). Site-specific valves were used. 